\title{
Quantum Stochastic Processes, Quantum Iterated Function Systems and Entropy
}

\author{
A. Baraviera; C. F. Lardizabal
}

\author{
A. O. Lopes and M. Terra Cunha
}

\begin{abstract}
We describe some basic results for Quantum Stochastic Processes and present some new results about a certain class of processes which are associated to Quantum Iterated Function Systems (QIFS). We discuss questions related to the Markov property and we present a definition of entropy which is induced by a QIFS. This definition is a natural generalization of the Shannon-Kolmogorov entropy from Ergodic Theory.
\end{abstract}

\section{Introduction}

We review and discuss some main properties of Quantum Stochastic Processes (see [6] [18] [20]) and present some new results about a certain class of processes which are associated to a Quantum Iterated Function System (QIFS). The concept of QIFS was introduced in the work [14], and it is a natural object in Quantum Information Theory.

We also present a definition of entropy which is suitable for the QIFS. This definition is a natural generalization of the Shannon-Kolmogorov entropy of Ergodic Theory. We describe a parallel between the classical Kolmogorov entropy and the one we present here, which is different from the one seen in [1].

The present definition of entropy is obtained by adapting the reasoning described in [5], [12] and [13] to the setting we present in this work. The main idea is to define this concept via the Ruelle operator and to avoid the use of partitions. Using this definition one can consider maximal pressure

Supported in part by CAPES and CNPq. 
density states. This formulation can be seen as a mini-max problem (see [5] [12] [13]). In [1] it is described some applications of the pressure problem.

Section 2 introduces basic notations and section 3 describes QSPs following [18]; section 4 and 5 describes Quantum Iterated Function Systems, following [14]. Section 6 is a brief digression on the Chapman-Kolmogorov equation and probability amplitudes. Section 7 defines probabilities measures and quantum stochastic processes induced by QIFS. Section 8 gives a definition of entropy induced by a QIFS and we make a few remarks on the variational problem of pressure.

Our work is inspired by results presented in [14] and [19]. We would like to thank these authors for supplying us with the corresponding references. Some other references related to the topic described here are [1] [2] [3] [4] [19].

This work is part of the thesis dissertation of C. F. Lardizabal in Prog. Pos-Grad. Mat. UFRGS (Brazil) [10].

\section{Notations}

We recall some basic notation which is used in Quantum Computing. For a comprehensive introduction to the subject, see [16]. Let $\mathcal{H}_{N}$ be a Hilbert space of finite dimension $N$. A state in a quantum system is described by $|\psi\rangle \in \mathcal{H}_{N}$. Such states are normalized, so we have $\langle\psi \mid \psi\rangle=$ 1. For any phase $\alpha$, we identify the elements $\left|\psi^{\prime}\right\rangle=e^{i \alpha}|\psi\rangle$ and $|\psi\rangle$, so we get the space of pure states, denoted by $\mathcal{P}_{N}$. Topologically, it is the complex projective space $\mathbb{C P}^{N-1}$ with the Fubini-Study metric, given by $D_{F S}(|\phi\rangle,|\psi\rangle):=\arccos |\langle\phi \mid \psi\rangle|$.

A qubit is a unit vector in a complex vector space of dimension 2

$$
|\psi\rangle=\alpha|0\rangle+\beta|1\rangle
$$

where $|\alpha|^{2}+|\beta|^{2}=1$. We can rewrite such equation as

$$
|\psi\rangle=e^{i \gamma}\left(\cos \frac{\theta}{2}|0\rangle+e^{i \phi} \sin \frac{\theta}{2}|1\rangle\right),
$$

where $\theta, \phi, \gamma$ are real numbers. As we are in projective space, the factor $e^{i \gamma}$ can be ignored, so we can write

$$
|\psi\rangle=\cos \frac{\theta}{2}|0\rangle+e^{i \phi} \sin \frac{\theta}{2}|1\rangle
$$

The numbers $\theta$ and $\phi$ define a point on the unit sphere, the Bloch sphere, which gives us an easy way to visualize the state of a qubit. 
Denote by $\rho^{*}$ the adjoint of $\rho: \mathcal{H}_{N} \rightarrow \mathcal{H}_{N}$. We say that $\rho: \mathcal{H}_{N} \rightarrow \mathcal{H}_{N}$ is hermitian if $\rho=\rho^{*}$. We say that a hermitian operator $P: \mathcal{H}_{N} \rightarrow \mathcal{H}_{N}$ is positive, denoting such fact by $P \geq 0$, if $\langle P v, v\rangle \geq 0, \forall v \in \mathcal{H}_{N}$.

Definition 1. A density operator (or density matrix) is an operator $\rho$ acting on $\mathcal{H}_{N}$, with $\rho=\rho^{*}, \rho \geq 0$ and $\operatorname{tr} \rho=1$. Denote by $\mathcal{M}_{N}$ the space of density operators.

If $|\psi\rangle \in \mathcal{H}_{N}$ is a state, denote its associated projection by $|\psi\rangle\langle\psi|$ (which defines an associated density matrix). We denote by $\{|0\rangle, \ldots, \mid N-$ $1\rangle\}$ the canonical orthonormal basis for $\mathcal{H}_{N}$. A density operator $\rho$ can always be written as

$$
\rho=\sum_{i=1}^{k} p_{i}\left|\psi_{i}\right\rangle\left\langle\psi_{i}\right|
$$

where the $p_{i}$ are positive numbers with $\sum_{i} p_{i}=1$, and $\left|\psi_{i}\right\rangle, i=1,2, \ldots N-1$, is an orthonormal basis. So the $\left|\psi_{i}\right\rangle$ are eigenvectors of $\rho$ with the $p_{i}$ as their respective eigenvalues.

If a density operator satisfies $\operatorname{tr}\left(\rho^{2}\right)=1$ then such operator is a projection and we say that $\rho$ represents a pure state. If $\rho$ is a nontrivial convex combination of projections then we say $\rho$ represents a mixed state; in this case we have $\operatorname{tr}\left(\rho^{2}\right)<1$. Also an operator is a density operator if and only if its trace equals 1 and if it is positive.

\section{A description of quantum stochastic process}

In this section the definitions and examples were taken from [18], where it is presented a definition of quantum stochastic process. We briefly describe some of the results obtained in that work.

Definition 2. A state space is a pair $(V, K)$, where

(1) $V$ is a real Banach space with norm $\|\cdot\|$.

(2) $K$ is a closed cone in $V$.

(3) If $u, v \in K$ then $\|u\|+\|v\|=\|u+v\|$

(4) If $u \in V$ and $\epsilon>0$ then there are $u_{1}, u_{2} \in K$ such that $u=u_{1}-u_{2}$ and $\left\|u_{1}\right\|+\left\|u_{2}\right\|<\|u\|+\epsilon$.

Definition 3. If $(V, K)$ is a state space then there is a unique positive linear functional $\tau: V \rightarrow \mathbb{R}$ such that $\tau(u)=\|u\|$ if $u \in K$, and $\tau(u) \leq\|u\|$ if $u \in V$. We say that $u \in K$ is a state if $\tau(u)=1$.

Example 1. Let $\mathcal{H}$ be a finite dimensional Hilbert space and let $V$ be the space of hermitian operators in $\mathcal{H}$. Let $K$ be the set of positive operators in $V$. In this case we have $\tau(B)=\operatorname{tr}(B)$ for all $B$ operator in $V$. 
Definition 4. A phase space is a measurable space $(\Omega, \Sigma)$ where $\Omega$ represents the set of all possible results for a measurement and $\Sigma$ is a $\sigma$-algebra of subsets of $\Omega$.

Let $V^{*}$ be the dual space of $V$. We introduce a partial order on $V^{*}$ by defining $\phi \geq \psi$ if $\phi(u) \geq \psi(u)$, for all $u \in K$.

Definition 5. An effect is a mapping $\phi \in V^{*}$ such that $0 \leq \phi \leq \tau$. We denote the space of effects by $\mathcal{E} \subset V^{*}$.

Definition 6. We say that $x: \Sigma \rightarrow \mathcal{E}$ is an observable if $x$ is a measure taking values on the space of effects, such that $x(\Omega)=\tau$.

If $E \in \Sigma, u \in K$ and $\tau(u)=1$ then $x(E) u$ can be interpreted as the probability that the result of the measurement of the physical quantity represented by $x$, prepared in the state $u$, belongs to the set $E$. In the case of quantum mechanics in Hilbert space, effects can be identified with bounded operators $A$ such that $0 \leq A \leq 1$ by the formula $\phi_{A}(W)=\operatorname{tr}(A W)$.

Definition 7. An operation is a positive linear operator $T: V \rightarrow V$ satisfying $0 \leq \tau(T u) \leq \tau(u)$ for all $u \in K$. The space of operations will be denoted by $\mathcal{O}$.

Definition 8. An operator valued measure, or an $O V M$ on a phase space is a map $\mathcal{I}: \Sigma \rightarrow \mathcal{O}$ such that if $\left\{E_{n}\right\}$ is a sequence of disjoint sets in $\Sigma$, then $\mathcal{I}\left(\cup E_{n}\right)=\sum \mathcal{I}\left(E_{n}\right)$.

Definition 9. Let $\mathcal{I}: \Sigma \rightarrow \mathcal{O}$ be an $O V M$, then we say that $\mathcal{I}$ is an instrument if

$$
\tau(\mathcal{I}(\Omega) u)=\tau(u), \forall u \in V .
$$

We interpret such notion in the following way. Let $\mathcal{I}$ be an instrument, $E \in \Sigma, u \in K$. If $u$ is the state of the system before the measurement and if $\mathcal{I}$ determines a value in $E$ then the resulting state is given by

$$
\frac{\mathcal{I}(E) u}{\tau(\mathcal{I}(E) u)}
$$

Note that for each instrument $\mathcal{I}$, there is a unique observable $x_{\mathcal{I}}: \Sigma \rightarrow \mathcal{E}$ such that $\tau(\mathcal{I}(E) u)=x_{\mathcal{I}}(E) u, E \in \Sigma, u \in K$. Also, it is possible that two instruments correspond to the same observable [18].

The following are examples of instruments: 
Example 2. Let $\mathcal{H}$ be a Hilbert space, and let $\mathcal{F}(\mathcal{H})$ be the space of hermitian operators $A$ in $\mathcal{H}$ such that

$$
\sum_{k \in \mathbb{N}}\left\langle e_{k}, A e_{k}\right\rangle<\infty
$$

and have the same value in any orthonormal base $\left\{e_{k}\right\}_{k \in \mathbb{N}}$ for $\mathcal{H}$. Let $\Omega=\{1, \ldots, N\}$, or $\Omega=\mathbb{N}$, let $\left\{P_{i}\right\}_{i \in \Omega}$ be a family of orthogonal projections such that $\sum_{i} P_{i}=I$. Define

$$
\begin{aligned}
& \mathcal{I}: \Sigma \rightarrow \mathcal{O} \\
& x_{\mathcal{I}}: \Sigma \rightarrow \mathcal{E}
\end{aligned}
$$

as

$$
\begin{aligned}
\mathcal{I}(E) \rho & :=\sum_{i \in E} P_{i} \rho P_{i}, \\
x_{\mathcal{I}}(E) \rho & :=\sum_{i \in E} \tau\left(P_{i} \rho\right),
\end{aligned}
$$

for all $E \subset \Omega$ and $\rho \in \mathcal{F}(\mathcal{H})$.

Example 3. Let $\mathcal{H}$ be a Hilbert space, $\Omega$ a topological space, $\Sigma$ a $\sigma$ algebra for $\Omega$ and $m$ a measure on $(\Omega, \Sigma)$. Let $\left\{P_{a}\right\}_{a \in \Omega}$ be a family of projections on $\mathcal{H}$, such that the mapping $a \rightarrow P_{a}$ is strongly continuous and $\int_{\Omega} P_{a} d m(a)=I$. Then define

$$
\begin{aligned}
& \mathcal{I}: \Sigma \rightarrow \mathcal{O} \\
& x_{\mathcal{I}}: \Sigma \rightarrow \mathcal{E}
\end{aligned}
$$

as

$$
\begin{aligned}
\mathcal{I}(E) \rho & :=\int_{E} P_{a} \rho P_{a} d m(a) \\
x_{\mathcal{I}}(E) \rho & :=\int_{E} \tau\left(P_{a} \rho\right) d m(a),
\end{aligned}
$$

for all $E \subset \Omega$ e $\rho \in \mathcal{F}(\mathcal{H})$.

Example 4. Let $X$ be a locally compact Hausdorff space, $V$ the space of the countably additive functions on the Borel $\sigma$-algebra $\mathcal{B}(X)$ for $X$ endowed with the norm of total variation. Let $K$ be the set of nonnegative measures on $V$. Let $(\Omega, \Sigma)=(X, \mathcal{B}(X))$. Then

$$
\mathcal{I}(E) \mu(A)=\mu(A \cap E)
$$


for $\mu \in V, A, E \in \Sigma$ is an instrument, called the sharp classical measurement and the corresponding observable is

$$
x_{\mathcal{I}}(E) \mu=\mu(E)
$$

Definition 10. Following [18], a Quantum Stochastic Process, QSP, is an arbitrary family of instruments $\left\{\mathcal{I}_{t}\right\}_{t \in \mathcal{J}}$. Let $\mathcal{J}=\mathbb{Z}$ or $\mathcal{J}=\mathbb{R}$ for discrete or continuous time, respectively.

The finite dimensional distributions of the process are measures $\mu_{t_{0}, \ldots, t_{n-1}}^{u}$ defined in $\left(\Omega^{n}, B\left(\Omega^{n}\right)\right)$ as being the natural extensions of the functions given by

$\mu_{t_{0}, \ldots, t_{n-1}}^{u}\left(E_{0} \times \cdots \times E_{n-1}\right)=\tau\left(\left(\mathcal{I}_{t_{n-1}}\left(E_{n-1}\right) \circ \mathcal{I}_{t_{n-2}}\left(E_{n-2}\right) \circ \cdots \circ \mathcal{I}_{t_{0}}\left(E_{0}\right)\right) u\right)$

where $n \in \mathbb{N}, t_{0} \leq \cdots \leq t_{n-1}, t_{i} \in \mathcal{J}, u \in V$ and $E_{0}, \ldots, E_{n-1} \in \Sigma$. The meaning of such expression is the following: $\mu_{t_{0}, \ldots, t_{n-1}}^{u}\left(E_{0} \times \cdots \times E_{n-1}\right)$ is the joint probability that successive measurements of the system by the instruments $\mathcal{I}_{0}, \ldots, \mathcal{I}_{n-1}$ in the moments $t_{0}, \ldots, t_{n-1}$ produce values in $E_{0}, \ldots, E_{n-1}$, when the pre-measurement state is $u$.

A probability transition is a function $P: \Omega \times \Sigma \rightarrow \mathbb{R}$ such that $P(\cdot, E)$ is measurable for all $E \in \Sigma$ and $P(x, \cdot)$ is a probability measure for all $x \in \Omega$.

Definition 11. We say that a QSP is Markov if there exists a family of probability transitions $\left\{P_{s, t}\right\}_{s<t}$ such that

$$
\begin{gathered}
\mu_{t_{0}, \ldots, t_{n-1}}^{u}\left(E_{0} \times \cdots \times E_{n-1}\right) \\
=\int_{E_{0}} \int_{E_{1}} \cdots \int_{E_{n}} P_{t_{n-1}, t_{n}}\left(y_{n-1}, d y_{n}\right) \cdots P_{t_{0}, t_{1}}\left(y_{0}, d y_{1}\right) \mu_{t_{0}}^{u}\left(d y_{0}\right)
\end{gathered}
$$

for all $t_{0}<\cdots<t_{n}, t_{i} \in \mathcal{J}, u \in V, E_{0}, \ldots, E_{n} \in \Sigma$. A Markov QSP is homogeneous if the probability transitions $P_{s, t}$ depend only on the difference $t-s$.

Remark In contrast with the classic theory of stochastic processes, the probability transitions of a Markov QSP do not satisfy in general the Chapman-Kolmogorov equation. 
Definition 12. Let $\mathcal{I}$ be an instrument. Assume that between the measurements the system evolves and its evolution is described by a group $\left\{T_{t}\right\}_{t \in \mathcal{J}}$ of isometric automorphisms of $V$. Then define the $Q S P\left\{\mathcal{I}_{t}\right\}_{t \in \mathcal{J}}$, where

$$
\mathcal{I}_{t}(E)=T_{t}^{-1} \circ \mathcal{I}(E) \circ T_{t}
$$

is called a transformed instrument. For simplicity, we can choose $\mathcal{J}=$ $\mathbb{Z}$ so $T_{n}=T^{n}$ and we denote such process by $\mathcal{C}(T, \mathcal{I})$.

Now we show an example of a Markov QSP.

Example 5. Let $\mathcal{I}$ be the instrument given in example 4 and let $\Theta: X \rightarrow X$ be a measurable map. Then $\Theta$ generates an automorphism $T_{\Theta}: V \rightarrow V$ by

$$
T_{\Theta}(\mu)(A)=\mu\left(\Theta^{-1}(A)\right), \quad \mu \in V, A \in B(X)
$$

Then we can show that $\mathcal{C}\left(T_{\Theta}, \mathcal{I}\right)$ is a homogeneous Markov QSP and its transition probability is given by

$$
P(x, E)=\mathcal{X}_{E}(\Theta x), \quad x \in X, E \in B(X)
$$

\section{Quantum IFS}

This section follows [14]. We begin with a few definitions.

Definition 13. Let $G_{i}: \mathcal{M}_{N} \rightarrow \mathcal{M}_{N}, p_{i}: \mathcal{M}_{N} \rightarrow[0,1], i=1, \ldots, k$ and such that $\sum_{i} p_{i}(\rho)=1$. We call

$$
\mathcal{F}_{N}=\left\{\mathcal{M}_{N}, G_{i}, p_{i}: i=1, \ldots, k\right\}
$$

$a$ Quantum Iterated Function System (QIFS).

Definition 14. A QIFS is homogeneous if $p_{i}$ and $G_{i} p_{i}$ are affine mappings, $i=1, \ldots, k$.

Suppose that the QIFS considered is such that there are $V_{i}$ and $W_{i}$ linear maps, $i=1, \ldots, k$, with $\sum_{i=1}^{k} W_{i}^{*} W_{i}=I$ such that

$$
G_{i}(\rho)=\frac{V_{i} \rho V_{i}^{*}}{\operatorname{tr}\left(V_{i} \rho V_{i}^{*}\right)}
$$

and

$$
p_{i}(\rho)=\operatorname{tr}\left(W_{i} \rho W_{i}^{*}\right)
$$

Then we have that a QIFS is homogeneous if $V_{i}=W_{i}, i=1, \ldots, k$. Now we can define a Markov operator $P: \mathcal{M}\left(\mathcal{M}_{N}\right) \rightarrow \mathcal{M}\left(\mathcal{M}_{N}\right)$,

$$
(P \mu)(B)=\sum_{i=1}^{k} \int_{G_{i}^{-1}(B)} p_{i}(\rho) d \mu(\rho),
$$


where $\mathcal{M}\left(\mathcal{M}_{N}\right)$ denotes the space of probability measure over $\mathcal{M}_{N}$. We also define $\Lambda: \mathcal{M}_{N} \rightarrow \mathcal{M}_{N}$,

$$
\Lambda(\rho):=\sum_{i=1}^{k} p_{i}(\rho) G_{i}(\rho)
$$

If the QIFS considered is homogeneous then

$$
\Lambda(\rho)=\sum_{i} V_{i} \rho V_{i}^{*}
$$

We say that $\rho \in \mathcal{M}_{N}$ is the integral of a mapping $f: \mathcal{M}_{N} \rightarrow \mathcal{M}_{N}$, denoted by

if

$$
\rho:=\int_{\mathcal{M}_{N}} f d \mu
$$

for all $l \in \mathcal{M}_{N}^{*}$.

$$
l(\rho)=\int_{\mathcal{M}_{N}} l \circ f d \mu
$$

Theorem 1. A mixed state $\rho_{0}$ is $\Lambda$-invariant, if and only if,

$$
\rho_{0}=\int_{\mathcal{M}_{N}} \rho d \mu(\rho)
$$

for some P-invariant measure $\mu$.

For the proof, see [14], [19].

In order to define hyperbolic QIFS, we have to specify a distance on the space of mixed states. The following are a few possibilities:

$$
\begin{gathered}
D_{1}\left(\rho_{1}, \rho_{2}\right)=\sqrt{\operatorname{tr}\left[\left(\rho_{1}-\rho_{2}\right)^{2}\right]} \\
D_{2}\left(\rho_{1}, \rho_{2}\right)=\operatorname{tr} \sqrt{\left(\rho_{1}-\rho_{2}\right)^{2}} \\
D_{3}\left(\rho_{1}, \rho_{2}\right)=\sqrt{2\left\{1-\operatorname{tr}\left[\left(\rho_{1}^{1 / 2} \rho_{2} \rho_{1}^{1 / 2}\right)^{1 / 2}\right]\right\}}
\end{gathered}
$$

Such metrics generate the same topology on $\mathcal{M}_{N}$. Considering the space of mixed states with one of those metrics we can make the following definition.

Definition 15. We say a QIFS is hyperbolic if the quantum maps $G_{i}$ are contractions with respect to one of the distances on $\mathcal{M}_{N}$ and if the maps $p_{i}$ are Hölder-continuous and positive.

Proposition 1. [14] [19] If a QIFS (15) is homogeneous and hyperbolic then the associated Markov operator admits a unique invariant measure $\mu$. Such invariant measure determines a unique $\Lambda$-invariant state $\rho \in \mathcal{M}_{N}$, given by (19). 


\section{Examples of QIFS}

Example 6. $\Omega=\mathcal{M}_{N}, k=2, p_{1}=p_{2}=1 / 2, G_{1}(\rho)=U_{1} \rho U_{1}^{*}, G_{2}(\rho)=$ $U_{2} \rho U_{2}^{*}$. The normalized identity matrix $\rho_{*}=I / N$ is $\Lambda$-invariant, for any choice of unitary $U_{1}$ and $U_{2}$. Note that we can write

$$
\rho_{*}=\int_{\mathcal{M}_{N}} \rho d \mu(\rho)
$$

where the measure $\mu$, uniformly distributed over $\mathcal{P}_{N}$, is $P$-invariant.

Example 7. Let $\Omega=\mathcal{M}_{N}, k=2, p_{1}=p_{2}=1 / 2, G_{1}(\rho)=\left(\rho+2 \rho_{1}\right) / 3$, $G_{2}(\rho)=\left(\rho+2 \rho_{2}\right) / 3$, where we choose the projectors $\rho_{1}=|1\rangle\langle 1|$ and $\rho_{2}=|2\rangle\langle 2|$ so that they are orthogonal. Since $G_{1}$ and $G_{2}$ are contractions with Lipschitz constant equal to $1 / 3$, this QIFS is hyperbolic and so there is a unique invariant measure.

Recall that a mapping $\Lambda$ is completely positive (CP), if $\Lambda \otimes I$ is positive for any extension of the original Hilbert space $\mathcal{H}_{N} \rightarrow \mathcal{H}_{N} \otimes \mathcal{H}_{E}$. We know that every trace preserving $\mathrm{CP}$ map can be represented (in a nonunique way) in the Stinespring-Kraus form

$$
\Lambda(\rho)=\sum_{j=1}^{k} V_{j} \rho V_{j}^{*}, \quad \sum_{j=1}^{k} V_{j}^{*} V_{j}=1,
$$

where the $V_{j}$ are linear operators. Besides, if $\sum_{j=1}^{k} V_{j} V_{j}^{*}=I$ then $\Lambda(I / N)=$ $I / N$ and $\Lambda$ will be called unital. This is the case if each of the $V_{j}$ is normal, that is, if $V_{j} V_{j}^{*}=V_{j}^{*} V_{j}$. Note that by writing $G_{i}(\rho)=U_{i} \rho U_{i}^{*}$, we have that example 6 is contained in this class of QIFS. We call such QIFS unitary. For a unitary QIFS we have that $\rho_{*}$ is an invariant state for $\Lambda_{U}$ and also that $\delta_{\rho_{*}}$ is invariant for the Markov operator $P_{U}$ induced by this QIFS.

Definition 16. We say that unitary matrices of same dimension are common block diagonal if they are block diagonal in the same base and with the same blocks.

The proof of the following lemma is presented in [14].

Proposition 2. Assume that $p_{i}, i=1, \ldots, k$ are strictly positive. The the maximally mixed state $\rho_{*}$ is the unique invariant state for the operator $\Lambda_{U}$ if and only if the unitary operators $U_{i}, i=1, \ldots, k$ are not common block diagonal. 
Example 8. Let $\Omega=\mathcal{P}_{2}, U_{1}=I, U_{2}=\sigma_{1}, U_{3}=\sigma_{2}, U_{4}=\sigma_{3}, p_{1}=1-p$, $p_{2}=p_{3}=p_{4}=p / 3>0$, where $\sigma_{1}, \sigma_{2}, \sigma_{3}$ are the Pauli matrices. Since such matrices are not common block diagonal the maximally mixed state $\rho_{*}$ is the unique invariant state for the mapping below, called a quantum depolarizing channel [14]:

$$
\Lambda_{U}(\rho)=\sum p_{i} U_{i} \rho U_{i}^{*}=(1-p) \rho+\frac{p}{3}\left(\sigma_{1} \rho \sigma_{1}+\sigma_{2} \rho \sigma_{2}+\sigma_{3} \rho \sigma_{3}\right) .
$$

Example 9. Let $\Omega=\mathcal{P}_{2}, p_{1}=1-p, p_{2}=p$,

$$
\begin{gathered}
U_{1}=\exp \left(-i H_{0} T / \hbar\right) \\
U_{2}=\exp \left(-\frac{i}{\hbar}\left(H_{0} T+\int_{0}^{T} V(t) d t\right)\right)
\end{gathered}
$$

where $V(t)=V(t+T)$. The maximally mixed state $\rho_{*}=I / 2$ is an invariant state for the operator $\Lambda_{U}$ corresponding to this QIFS. For a generic perturbation $V$, matrices $U_{1}$ and $U_{2}$ are not common block diagonal so $\rho_{*}$ is the unique invariant state for $\Lambda_{U}$.

\section{On certain probability and amplitude calculations}

We begin with a brief digression on the Chapman-Kolmogorov equation. Let $X=\left\{X_{n}\right\}$ be a sequence of measurable functions. Suppose that

$$
P\left(X_{n+1}=j \mid X_{n}=i\right)=P\left(X_{1}=j \mid X_{0}=i\right)
$$

for all $n, i, j$. Suppose that $X$ takes values on a finite set $S$. Define the matrix $P=\left(p_{i j}\right)$ of order $|S|$, with entries

$$
p_{i j}=P\left(X_{n+1}=j \mid X_{n}=i\right)
$$

Define the matrix of $n$ transitions $P_{n}=\left(p_{i j}(n)\right)$, where

$$
p_{i j}(n)=P\left(X_{m+n}=j \mid X_{m}=i\right)
$$

Also suppose that it is a Markov chain, that is

$$
\begin{gathered}
P\left(X_{n}=x_{n} \mid X_{0}=x_{0}, X_{1}=x_{1}, \ldots, X_{n-1}=x_{n-1}\right)= \\
P\left(X_{n}=x_{n} \mid X_{n-1}=x_{n-1}\right)
\end{gathered}
$$

for all $n \geq 1$, and $x_{0}, \ldots, x_{n} \in S$.

By using the fact that for any events $A_{1}, A_{2}, A_{3}$, we have

$$
P\left(A_{1} \cap A_{2} \mid A_{3}\right)=P\left(A_{1} \mid A_{2} \cap A_{3}\right) P\left(A_{2} \mid A_{3}\right)
$$


we can write

$$
\begin{aligned}
p_{i j}(m+n) & =P\left(X_{m+n}=j \mid X_{0}=i\right)=\sum_{k} P\left(X_{m+n}=j, X_{m}=k \mid X_{0}=i\right) \\
& =\sum_{k} P\left(X_{m+n}=j \mid X_{m}=k\right) P\left(X_{m}=k \mid X_{0}=i\right)
\end{aligned}
$$

So

$$
p_{i j}(m+n)=\sum_{k} p_{i k}(m) p_{k j}(n)
$$

which is the Chapman-Kolmogorov equation. We are interested in studying quantum stochastic processes and in obtaining an adequate definition to what we will call a Markov quantum stochastic process. First we recall that in the previous section we have presented a description [18] of Markov QSP in which the Chapman-Kolmogorov do not hold in general. This fact can be seen as the general rule for quantum processes (but see [8] for different settings).

In algebraic terms, we can argue that the deduction of (23) above is not valid for quantum processes because of equation (21). Since we have to take in consideration the interference between measurements, the problem of understanding how probability measures work in a quantum setting is a basic question. In quantum mechanics we could in principle consider a probability space $(\Omega, \Lambda, \mu)$ such as in classic measure theory. However, we have that $\Lambda$ is a $\sigma$-algebra and $\mu$ is a measure on $\Lambda$ only when we are restricted to a single measurement. When we perform several measurements interference effects occur and so we are no longer considering a problem on classic probability [7]. Results of more general nature are presented in [9].

We can think that interference occurs because, in contrast to classic probability measures, which can be quite arbitrary, quantum probability measures are obtained in a very specific way. In quantum mechanics we have an amplitude function $a: \Omega \rightarrow \mathbb{C}$, and if $B \in \Lambda$, we define the amplitude of $B$ as

$$
A(B)=\sum_{\omega \in B} a(\omega)
$$

and we define the probability that $B$ occurs as

$$
\mu(B)=|A(B)|^{2}
$$

Let us describe a few more details on this point. For more on the subject, see for instance [7]. Let $\Omega$ be a nonempty set and let $a: \Omega \rightarrow \mathbb{C}$. We say that $\omega \in \Omega$ is a sample point and the map $a$ is a probability amplitude, and $(\Omega, f)$ is called a quantum probability space. A set $A \subset \Omega$ is summable 
if $\sum_{\omega \in \Omega}|a(\omega)|^{2}<\infty$ and we denote the collection of summable sets by $\Sigma_{0}$. Now define $A: \Sigma_{0} \rightarrow \mathbb{C}$ as $A(\emptyset)=0$ and

$$
A(B):=\sum_{\omega \in \Omega} a(\omega)
$$

We say that $A(B)$ is the amplitude of $B$. Now define

$$
A\left(B_{1} \mid B_{2}\right):=\frac{A\left(B_{1} \cap B_{2}\right)}{A\left(B_{2}\right)}
$$

if $A\left(B_{2}\right) \neq 0$ and equal to zero, otherwise. In the case that $A\left(B_{2}\right) \neq 0$, we have that $A\left(\cdot \mid B_{2}\right)$ is a complex measure on $P(\Omega)$, with $A\left(\Omega \mid B_{2}\right)=1$. We say that $A\left(B_{1} \mid B_{2}\right)$ is the conditional amplitude of $B_{1}$, given $B_{2}$. Note that $A(B)=0$ does not imply $A(B \cap C)=0$ [7]. Because of that, formulas of the kind $A(B \cap C)=A(B) A(C \mid B)$ might not be true when $A(B)=0$. However, when the conditioning sets have a nonzero amplitude, we have the formula

$$
A\left(B_{1} \cap \cdots \cap B_{n}\right)=A\left(B_{1}\right) A\left(B_{2} \mid B_{1}\right) A\left(B_{3} \mid B_{1} \cap B_{2}\right) \cdots A\left(B_{n} \mid B_{1} \cap \cdots \cap B_{n-1}\right)
$$

which is the amplitude counterpart for equation (21). Define the matrix $A=\left(a_{i j}\right)$, where $a_{i j}=A\left(X_{n+1}=j \mid X_{n}=i\right)$. Now suppose that the chain $\left\{X_{n}\right\}_{n \in \mathbb{N}}$ is quantum Markov, that is,

$$
\begin{gathered}
A\left(X_{n}=x_{n} \mid X_{0}=x_{0}, X_{1}=x_{1}, \ldots, X_{n-1}=x_{n-1}\right)= \\
A\left(X_{n}=x_{n} \mid X_{n-1}=x_{n-1}\right)
\end{gathered}
$$

for all $n \geq 1, x_{0}, \ldots, x_{n} \in S$. So in a way which is similar to what we did for probabilities, define the matrix of $n$ transitions $A_{n}=\left(a_{i j}(n)\right)$, where $a_{i j}(n)=A\left(X_{m+n}=j \mid X_{m}=i\right)$ and we get

$$
a_{i j}(m+n)=\sum_{k} a_{i k}(m) a_{k j}(n)
$$

so we have that $A_{m+n}=A_{m} A_{n}$ and $A_{n}=A^{n}$.

\section{Probability measures induced by QIFS}

In this section we present some new results. Consider a Hilbert space $\mathcal{H}$ of dimension $N=2$. Let $q_{1}, q_{2} \in \mathbb{R}$ and also

$$
V_{1}=\left(\begin{array}{cc}
\sqrt{p_{11}} & \sqrt{p_{12}} \\
0 & 0
\end{array}\right), \quad V_{2}=\left(\begin{array}{cc}
0 & 0 \\
\sqrt{p_{21}} & \sqrt{p_{22}}
\end{array}\right), \quad \rho=\left(\begin{array}{ll}
\rho_{1} & \rho_{2} \\
\rho_{3} & \rho_{4}
\end{array}\right)
$$

We would like to obtain the fixed points for

$$
\mathcal{L}(\rho)=q_{1} V_{1} \rho V_{1}^{*}+q_{2} V_{2} \rho V_{2}^{*}
$$


Then

$$
q_{1} V_{1} \rho V_{1}^{*}+q_{2} V_{2} \rho V_{2}^{*}=\rho
$$

implies

$$
\begin{aligned}
& q_{1}\left[\left(\sqrt{p_{11}} \rho_{1}+\sqrt{p_{12}} \rho_{3}\right) \sqrt{p_{11}}+\left(\sqrt{p_{11}} \rho_{2}+\sqrt{p_{12}} \rho_{4}\right) \sqrt{p_{12}}\right]=\rho_{1} \\
& q_{2}\left[\left(\sqrt{p_{21}} \rho_{1}+\sqrt{p_{22}} \rho_{3}\right) \sqrt{p_{21}}+\left(\sqrt{p_{21}} \rho_{2}+\sqrt{p_{22}} \rho_{4}\right) \sqrt{p_{22}}\right]=\rho_{4}
\end{aligned}
$$

And (32) also implies that $\rho_{2}=\rho_{3}=0$, so we rewrite the system as

$$
\begin{aligned}
& q_{1}\left[\sqrt{p_{11}} \rho_{1} \sqrt{p_{11}}+\sqrt{p_{12}} \rho_{4} \sqrt{p_{12}}\right]=\rho_{1} \\
& q_{2}\left[\sqrt{p_{21}} \rho_{1} \sqrt{p_{21}}+\sqrt{p_{22}} \rho_{4} \sqrt{p_{22}}\right]=\rho_{4}
\end{aligned}
$$

or

$$
\begin{aligned}
& a \rho_{1}+f \rho_{4}=\rho_{1} \\
& g \rho_{1}+h \rho_{4}=\rho_{4}
\end{aligned}
$$

where

$$
a=q_{1} p_{11}, \quad f=q_{1} p_{12}, \quad g=q_{2} p_{21}, \quad h=q_{2} p_{22}
$$

We also get that

$$
\begin{aligned}
& \rho_{1}=\frac{f}{1-a} \rho_{4} \\
& \rho_{1}=\frac{1-h}{g} \rho_{4}
\end{aligned}
$$

which is a restriction on the $q_{i}$, namely

$$
\frac{f}{1-a}=\frac{1-h}{g}
$$

Therefore the solution of (33) and (34) is

$$
\rho=\rho_{4}\left(\begin{array}{cc}
\frac{f}{1-a} & 0 \\
0 & 1
\end{array}\right)=\rho_{4}\left(\begin{array}{cc}
\frac{1-h}{g} & 0 \\
0 & 1
\end{array}\right)
$$

But $\rho_{1}+\rho_{4}=1$ implies

$$
\rho=\left(\begin{array}{cc}
\frac{q_{1} p_{12}}{q_{1} p_{12}-p_{1} p_{11}+1} & 0 \\
0 & \frac{1-q_{1} p_{11}}{q_{1} p_{12}-q_{1} p_{11}+1}
\end{array}\right)=\left(\begin{array}{cc}
\frac{1-q_{2} p_{22}}{1-q_{2} p_{22}+q_{2} p_{21}} & 0 \\
0 & \frac{q_{2} p_{21}}{1-q_{2} p_{22}+q_{2} p_{21}}
\end{array}\right)
$$

Now assume that

$$
P=\left(\begin{array}{ll}
p_{11} & p_{12} \\
p_{21} & p_{22}
\end{array}\right)
$$


is column stochastic, that is, we have $p_{i j} \geq 0$ and $\sum_{i} p_{i j}=1, i, j=1,2$. Let $\pi$ be such that $P \pi=\pi$. Such $\pi$ is given by

$$
\pi=\left(\frac{p_{12}}{p_{12}-p_{11}+1}, \frac{1-p_{11}}{p_{12}-p_{11}+1}\right)
$$

Compare (36) with (35). Then fix $q_{1}=q_{2}=1$, so we get that the nonzero entries of $\rho$ are equal to the entries of $\pi$. Such a choice for the $q_{i}$ is unique. In fact, comparing the $(i, i)$-th entry of $\rho$ with the $i$-th coordinate of $\pi$, we see that if there exists $q_{i}^{\prime}$ which make $\rho$ and $\pi$ equal (i.e., the diagonal entries of $\rho$ correspond to the entries of $\pi$ ), then

which implies

$$
\frac{q_{1} p_{12}}{q_{1} p_{12}-q_{1} p_{11}+1}=\frac{q_{1}^{\prime} p_{12}}{q_{1}^{\prime} p_{12}-q_{1}^{\prime} p_{11}+1},
$$

$$
\begin{aligned}
& q_{1}\left(q_{1}^{\prime} p_{12}-q_{1}^{\prime} p_{11}+1\right)=q_{1}^{\prime}\left(q_{1} p_{12}-q_{1} p_{11}+1\right) \\
\Rightarrow & q_{1} q_{1}^{\prime} p_{12}-q_{1} q_{1}^{\prime} p_{11}+q_{1}=q_{1} q_{1}^{\prime} p_{12}-q_{1} q_{1}^{\prime} p_{11}+q_{1}^{\prime}
\end{aligned}
$$

and when we cancel terms we get $q_{1}=q_{1}^{\prime}$. In a similar way

$$
\frac{1-q_{2} p_{22}}{1-q_{2} p_{22}+q_{2} p_{21}}=\frac{1-q_{2}^{\prime} p_{22}}{1-q_{2}^{\prime} p_{22}+q_{2}^{\prime} p_{21}}
$$

implies

$$
\begin{gathered}
\left(1-q_{2} p_{22}\right)\left(1-q_{2}^{\prime} p_{22}+q_{2}^{\prime} p_{21}\right)=\left(1-q_{2}^{\prime} p_{22}\right)\left(1-q_{2} p_{22}+q_{2} p_{21}\right) \\
\Rightarrow 1-q_{2}^{\prime} p_{22}+q_{2}^{\prime} p_{21}-q_{2} p_{22}+q_{2} q_{2}^{\prime} p_{22}^{2}-q_{2} q_{2}^{\prime} p_{22} p_{21} \\
=1-q_{2} p_{22}+q_{2} p_{21}-q_{2}^{\prime} p_{22}+q_{2} q_{2}^{\prime} p_{22}^{2}-q_{2} q_{2}^{\prime} p_{22} p_{21}
\end{gathered}
$$

Then we get

$$
q_{2}^{\prime} p_{21}=q_{2} p_{21} \Rightarrow q_{2}^{\prime}=q_{2}
$$

and therefore the choice for $q_{1}$ and $q_{2}$ is unique.

Consider a homogeneous QIFS $\mathcal{F}=\left\{\mathcal{M}_{N}, F_{i}, p_{i}\right\}_{i=1, \ldots, k}$, where

$$
F_{i}(\rho)=\frac{V_{i} \rho V_{i}^{*}}{\operatorname{tr}\left(V_{i} \rho V_{i}^{*}\right)}
$$

where the $V_{i}$ are linear with $\sum_{i} V_{i}^{*} V_{i}=I$ and $p_{i}(\rho)=\operatorname{tr}\left(V_{i} \rho V_{i}^{*}\right)$. Then $\Lambda$ is written as

$$
\Lambda(\rho)=\sum_{i} p_{i} F_{i}=\sum_{i} V_{i} \rho V_{i}^{*}
$$

By simplicity we will assume that the quantum system considered can assume two states called 1 and 2 . 
We say that the pair $\left(\left\{X_{n}\right\}_{n \in \mathbb{N}}, \mu\right), X_{n}: \Omega \rightarrow\{1, \ldots, k\}$, is a Quantum Stochastic Process, QSP (homogeneous case), associated to the QIFS $\mathcal{F}$ whenever $\mu$ is defined as

$$
\mu\left(X_{1}=x_{1}, \ldots, X_{n}=x_{n}\right):=\operatorname{tr}\left(V_{x_{n}} V_{x_{n-1}} \cdots V_{x_{2}} V_{x_{1}} \rho_{0} V_{x_{1}}^{*} V_{x_{2}}^{*} \cdots V_{x_{n-1}}^{*} V_{x_{n}}^{*}\right)
$$

where $\rho_{0} \in \mathcal{M}_{N}$ is any density operator. The operator $\rho_{0}$ is a pre-measure ment state, that is, we have a quantum system and we prepare $\rho_{0}$ as being its initial state (for a similar treatment to a sequence of measurements, see the definition of finite dimensional distributions in section 3).

So we can define for any $r$,

$$
\mu\left(X_{r}=x_{r} \mid X_{r-1}=x_{r-1}\right)=\frac{\operatorname{tr}\left(V_{x_{r}} V_{x_{r-1}} \rho_{0} V_{x_{r-1}}^{*} V_{x_{r}}^{*}\right)}{\operatorname{tr}\left(V_{x_{r-1}} \rho_{0} V_{x_{r-1}}^{*}\right)}
$$

Definition 17. We say that a $Q S P$ is Markov if

$$
\mu\left(X_{n}=x_{n} \mid X_{1}=x_{1}, \ldots, X_{n-1}=x_{n-1}\right)=\mu\left(X_{n}=x_{n} \mid X_{n-1}=x_{n-1}\right)
$$

Remark The condition $\sum_{i} V_{i}^{*} V_{i}=I$ is enough to show that the measure of a partition of cylinder sets equals 1 . For instance, for two states 1 and 2 , for $k=2$ and writing

$$
\mu(\overline{i j}):=\mu\left(X_{1}=i, X_{2}=j\right),
$$

we have

$$
\begin{gathered}
\mu(\overline{11})+\mu(\overline{12})+\mu(\overline{21})+\mu(\overline{22}) \\
=\operatorname{tr}\left(V_{1} V_{1} \rho V_{1}^{*} V_{1}^{*}\right)+\operatorname{tr}\left(V_{2} V_{1} \rho V_{1}^{*} V_{2}^{*}\right)+\operatorname{tr}\left(V_{1} V_{2} \rho V_{2}^{*} V_{1}^{*}\right)+\operatorname{tr}\left(V_{2} V_{2} \rho V_{2}^{*} V_{2}^{*}\right) \\
=\operatorname{tr}\left(V_{1}^{*} V_{1}\left[V_{1} \rho V_{1}^{*}\right]\right)+\operatorname{tr}\left(V_{2}^{*} V_{2}\left[V_{1} \rho V_{1}^{*}\right]\right)+\operatorname{tr}\left(V_{1}^{*} V_{1}\left[V_{2} \rho V_{2}^{*}\right]\right)+\operatorname{tr}\left(V_{2}^{*} V_{2}\left[V_{2} \rho V_{2}^{*}\right]\right) \\
=\operatorname{tr}\left(\left(V_{1}^{*} V_{1}+V_{2}^{*} V_{2}\right)\left[V_{1} \rho V_{1}^{*}\right]\right)+\operatorname{tr}\left(\left(V_{1}^{*} V_{1}+V_{2}^{*} V_{2}\right)\left[V_{2} \rho V_{2}^{*}\right]\right) \\
=\operatorname{tr}\left(V_{1} \rho V_{1}^{*}\right)+\operatorname{tr}\left(V_{2} \rho V_{2}^{*}\right)=\operatorname{tr}\left(\left(V_{1}^{*} V_{1}+V_{2}^{*} V_{2}\right) \rho\right)=1
\end{gathered}
$$

However, we note that there exist examples in which we can show that the measure of a partition of cylinder sets equals 1 even if we do not suppose that $\sum_{i} V_{i}^{*} V_{i}=I$. This happens, for instance, in the following construction involving stochastic matrices.

Let us consider the particular case in which the operator $\rho_{0} \in \mathcal{M}_{N}$, given in the definition of QSP is a fixed point for $\Lambda(\rho)=\sum_{i=1}^{k} V_{i} \rho V_{i}^{*}$ induced by the QIFS $\mathcal{F}$. 
Suppose that $V_{1}$ and $V_{2}$ are defined by (31). Suppose that the matrix $P=\left(p_{i j}\right)$ is column stochastic and that we have $\pi$ such that $P \pi=\pi$. For instance we have

$$
\mu\left(X_{1}=1, X_{2}=2\right)=\operatorname{tr}\left(V_{2} V_{1} \rho_{0} V_{1}^{*} V_{2}^{*}\right)=p_{21}\left(p_{11} \rho_{11}+p_{12} \rho_{22}\right)=p_{21} \rho_{11}
$$

because with the choice of $V_{i}$ we made, we have that the nonzero entries of $\rho_{0}$ correspond to the entries of $\pi$. So we can interpret $p_{i j}$ as being

$$
p_{i j}=\mu\left(X_{2}=j \mid X_{1}=i\right)
$$

In a similar way,

$$
\mu\left(X_{1}=2, X_{2}=1\right)=\operatorname{tr}\left(V_{1} V_{2} \rho_{0} V_{2}^{*} V_{1}^{*}\right)=p_{12} \rho_{22}
$$

and

$$
\mu\left(X_{1}=1, X_{2}=2, X_{3}=1\right)=\operatorname{tr}\left(V_{1} V_{2} V_{1} \rho_{0} V_{1}^{*} V_{2}^{*} V_{1}^{*}\right)=p_{12} p_{21} \rho_{11}
$$

Remark A simple calculation shows that with the $V_{i}$ given by (31) we have that $\sum_{i} V_{i}^{*} V_{i} \neq I$. However, we still have that

$$
\mu(\overline{11})+\mu(\overline{12})+\mu(\overline{21})+\mu(\overline{22})=1
$$

To prove that the choice (31) reduces to the classic case for any sequence, we use the following lemma.

Lemma 1. Suppose $N=2, k=2$. Then for every $m$, for $V_{i}$ given by (31) and $\rho_{0}$ corresponding to the stationary vector $\pi$ for $P$, we have that the product

has the form

$$
V_{x_{m}} V_{x_{m-1}} \cdots V_{x_{1}} \rho_{0} V_{x_{1}}^{*} V_{x_{2}}^{*} \cdots V_{x_{m}}^{*}
$$

$$
\left(\begin{array}{ll}
* & 0 \\
0 & 0
\end{array}\right) \text { ou }\left(\begin{array}{ll}
0 & 0 \\
0 & *
\end{array}\right)
$$

depending on whether $x_{m}=1$ or $x_{m}=2$, respectively.

Proof By induction. If $m=1$ then

$$
V_{1} \rho_{0} V_{1}^{*}=\left(\begin{array}{cc}
p_{11} \rho_{11}+p_{12} \rho_{22} & 0 \\
0 & 0
\end{array}\right)
$$

and

$$
V_{2} \rho_{0} V_{2}^{*}=\left(\begin{array}{cc}
0 & 0 \\
0 & p_{21} \rho_{11}+p_{22} \rho_{22}
\end{array}\right)
$$

Suppose the lemma valid for $m$, we consider the product

$$
V_{x_{m+1}} V_{x_{m}} \cdots V_{x_{1}} \rho_{0} V_{x_{1}}^{*} V_{x_{2}}^{*} \cdots V_{x_{m}}^{*} V_{x_{m+1}}^{*}
$$


Suppose $x_{m+1}=1$. Then a simple calculation shows that

$$
V_{1}\left(\begin{array}{cc}
* & 0 \\
0 & 0
\end{array}\right) V_{1}^{*} \text { e } V_{1}\left(\begin{array}{cc}
0 & 0 \\
0 & *
\end{array}\right) V_{1}^{*}
$$

has only one nonzero entry, namely the $(1,1)$-th entry. We proceed in a similar way for the case that $x_{m+1}=2$, that is

$$
V_{2}\left(\begin{array}{ll}
* & 0 \\
0 & 0
\end{array}\right) V_{2}^{*} \text { e } V_{2}\left(\begin{array}{ll}
0 & 0 \\
0 & *
\end{array}\right) V_{2}^{*}
$$

has only one nonzero entry, namely the $(2,2)$-th entry.

Proposition 3. If we set

$$
V_{1}=\left(\begin{array}{cc}
\sqrt{p_{11}} & \sqrt{p_{12}} \\
0 & 0
\end{array}\right), \quad V_{2}=\left(\begin{array}{cc}
0 & 0 \\
\sqrt{p_{21}} & \sqrt{p_{22}}
\end{array}\right)
$$

then

$$
\mu\left(X_{1}=x_{1}, X_{2}=x_{2}, \ldots, X_{n}=x_{n}\right)=p_{x_{n} x_{n-1}} p_{x_{n-1} x_{n-2}} \cdots p_{x_{3} x_{2}} p_{x_{2} x_{1}} \rho_{x_{1} x_{1}}
$$

where $\rho_{i j}$ denotes the $(i, j)$-th entry of $\rho_{0}$, eigenstate for $\Lambda(\rho)=\sum_{i} V_{i} \rho V_{i}^{*}$.

Proof We prove by induction. Suppose $n=1$. Then

$$
\begin{aligned}
& \mu\left(X_{1}=1\right)=\operatorname{tr}\left(V_{1} \rho_{0} V_{1}^{*}\right)=p_{11} \rho_{11}+p_{12} \rho_{22}=\rho_{11} \\
& \mu\left(X_{1}=2\right)=\operatorname{tr}\left(V_{2} \rho_{0} V_{2}^{*}\right)=p_{21} \rho_{11}+p_{22} \rho_{22}=\rho_{22}
\end{aligned}
$$

For the sake of clarity we also show the case $n=2$. We have, after some routine calculations that

$$
\begin{aligned}
& \mu\left(X_{1}=1, X_{2}=1\right)=\operatorname{tr}\left(V_{1} V_{1} \rho_{0} V_{1}^{*} V_{1}^{*}\right)=p_{11} \rho_{11} \\
& \mu\left(X_{1}=1, X_{2}=2\right)=\operatorname{tr}\left(V_{2} V_{1} \rho_{0} V_{1}^{*} V_{2}^{*}\right)=p_{21} \rho_{11} \\
& \mu\left(X_{1}=2, X_{2}=1\right)=\operatorname{tr}\left(V_{1} V_{2} \rho_{0} V_{2}^{*} V_{1}^{*}\right)=p_{12} \rho_{22} \\
& \mu\left(X_{1}=2, X_{2}=2\right)=\operatorname{tr}\left(V_{2} V_{2} \rho_{0} V_{2}^{*} V_{2}^{*}\right)=p_{22} \rho_{22}
\end{aligned}
$$

Now suppose the lemma holds for $n$, let us prove it for $n+1$.

First suppose $x_{n+1}=1$. Then

$$
\begin{gathered}
\mu\left(X_{1}=x_{1}, \ldots, X_{n}=x_{n}, X_{n+1}=1\right) \\
=\operatorname{tr}\left(V_{1} V_{x_{n}} \cdots V_{x_{2}} V_{x_{1}} \rho_{0} V_{x_{1}}^{*} V_{x_{2}}^{*} \cdots V_{x_{n-1}}^{*} V_{x_{n}}^{*} V_{1}^{*}\right)
\end{gathered}
$$

Using lemma 1, we have two cases. If $x_{n}=1$ then

$$
V_{x_{n}} \cdots V_{x_{2}} V_{x_{1}} \rho_{0} V_{x_{1}}^{*} V_{x_{2}}^{*} \cdots V_{x_{n-1}}^{*} V_{x_{n}}^{*}=\left(\begin{array}{cc}
* & 0 \\
0 & 0
\end{array}\right)
$$


and therefore

$V_{1} V_{x_{n}} \cdots V_{x_{2}} V_{x_{1}} \rho_{0} V_{x_{1}}^{*} V_{x_{2}}^{*} \cdots V_{x_{n-1}}^{*} V_{x_{n}}^{*} V_{1}^{*}=V_{1}\left(\begin{array}{cc}* & 0 \\ 0 & 0\end{array}\right) V_{1}^{*}=\left(\begin{array}{cc}* p_{11} & 0 \\ 0 & 0\end{array}\right)$

and so by taking the trace we get

$$
\begin{gathered}
\operatorname{tr}\left(V_{1} V_{x_{n}} \cdots V_{x_{2}} V_{x_{1}} \rho_{0} V_{x_{1}}^{*} V_{x_{2}}^{*} \cdots V_{x_{n-1}}^{*} V_{x_{n}}^{*} V_{1}^{*}\right) \\
\quad=p_{11} p_{1 x_{n-1}} p_{x_{n-1} x_{n-2}} \cdots p_{x_{3} x_{2}} p_{x_{2} x_{1}} \rho_{x_{1} x_{1}}
\end{gathered}
$$

In a similar way, if $x_{n}=2$,

$$
\begin{gathered}
V_{x_{n}} \cdots V_{x_{2}} V_{x_{1}} \rho_{0} V_{x_{1}}^{*} V_{x_{2}}^{*} \cdots V_{x_{n-1}}^{*} V_{x_{n}}^{*}=\left(\begin{array}{cc}
0 & 0 \\
0 & *
\end{array}\right) \\
V_{1} V_{x_{n}} \cdots V_{x_{2}} V_{x_{1}} \rho_{0} V_{x_{1}}^{*} V_{x_{2}}^{*} \cdots V_{x_{n-1}}^{*} V_{x_{n}}^{*} V_{1}^{*}=V_{1}\left(\begin{array}{cc}
0 & 0 \\
0 & *
\end{array}\right) V_{1}^{*}=\left(\begin{array}{cc}
* p_{12} & 0 \\
0 & 0
\end{array}\right)
\end{gathered}
$$

and taking the trace gives

$$
\begin{gathered}
\operatorname{tr}\left(V_{1} V_{x_{n}} \cdots V_{x_{2}} V_{x_{1}} \rho_{0} V_{x_{1}}^{*} V_{x_{2}}^{*} \cdots V_{x_{n-1}}^{*} V_{x_{n}}^{*} V_{1}^{*}\right) \\
\quad=p_{12} p_{2 x_{n-1}} p_{x_{n-1} x_{n-2}} \cdots p_{x_{3} x_{2}} p_{x_{2} x_{1}} \rho_{x_{1} x_{1}}
\end{gathered}
$$

Now we suppose $x_{n+1}=2$, and we proceed in an analogous way.

$$
\begin{gathered}
\mu\left(X_{1}=x_{1}, \ldots, X_{n}=x_{n}, X_{n+1}=2\right) \\
=\operatorname{tr}\left(V_{2} V_{x_{n}} \cdots V_{x_{2}} V_{x_{1}} \rho_{0} V_{x_{1}}^{*} V_{x_{2}}^{*} \cdots V_{x_{n-1}}^{*} V_{x_{n}}^{*} V_{2}^{*}\right)
\end{gathered}
$$

By lemma 1, we have two cases. If $x_{n}=1$ then

$$
V_{x_{n}} \cdots V_{x_{2}} V_{x_{1}} \rho_{0} V_{x_{1}}^{*} V_{x_{2}}^{*} \cdots V_{x_{n-1}}^{*} V_{x_{n}}^{*}=\left(\begin{array}{cc}
* & 0 \\
0 & 0
\end{array}\right)
$$

therefore

$V_{2} V_{x_{n}} \cdots V_{x_{2}} V_{x_{1}} \rho_{0} V_{x_{1}}^{*} V_{x_{2}}^{*} \cdots V_{x_{n-1}}^{*} V_{x_{n}}^{*} V_{2}^{*}=V_{2}\left(\begin{array}{cc}* & 0 \\ 0 & 0\end{array}\right) V_{2}^{*}=\left(\begin{array}{cc}* p_{21} & 0 \\ 0 & 0\end{array}\right)$

and taking the trace we get

$$
\begin{gathered}
\operatorname{tr}\left(V_{2} V_{x_{n}} \cdots V_{x_{2}} V_{x_{1}} \rho_{0} V_{x_{1}}^{*} V_{x_{2}}^{*} \cdots V_{x_{n-1}}^{*} V_{x_{n}}^{*} V_{2}^{*}\right) \\
\quad=p_{21} p_{1 x_{n-1}} p_{x_{n-1} x_{n-2}} \cdots p_{x_{3} x_{2}} p_{x_{2} x_{1}} \rho_{x_{1} x_{1}}
\end{gathered}
$$

Analogously if $x_{n}=2$

$$
\begin{gathered}
V_{x_{n}} \cdots V_{x_{2}} V_{x_{1}} \rho_{0} V_{x_{1}}^{*} V_{x_{2}}^{*} \cdots V_{x_{n-1}}^{*} V_{x_{n}}^{*}=\left(\begin{array}{cc}
0 & 0 \\
0 & *
\end{array}\right) \\
V_{2} V_{x_{n}} \cdots V_{x_{2}} V_{x_{1}} \rho_{0} V_{x_{1}}^{*} V_{x_{2}}^{*} \cdots V_{x_{n-1}}^{*} V_{x_{n}}^{*} V_{2}^{*}=V_{2}\left(\begin{array}{cc}
0 & 0 \\
0 & *
\end{array}\right) V_{2}^{*}=\left(\begin{array}{cc}
* p_{22} & 0 \\
0 & 0
\end{array}\right)
\end{gathered}
$$


and taking the trace

$$
\begin{gathered}
\operatorname{tr}\left(V_{2} V_{x_{n}} \cdots V_{x_{2}} V_{x_{1}} \rho_{0} V_{x_{1}}^{*} V_{x_{2}}^{*} \cdots V_{x_{n-1}}^{*} V_{x_{n}}^{*} V_{2}^{*}\right) \\
\quad=p_{22} p_{2 x_{n-1}} p_{x_{n-1} x_{n-2}} \cdots p_{x_{3} x_{2}} p_{x_{2} x_{1}} \rho_{x_{1} x_{1}}
\end{gathered}
$$

Corollary 1. The quantum stochastic process induced by

$$
V_{1}=\left(\begin{array}{cc}
\sqrt{p_{11}} & \sqrt{p_{12}} \\
0 & 0
\end{array}\right), \quad V_{2}=\left(\begin{array}{cc}
0 & 0 \\
\sqrt{p_{21}} & \sqrt{p_{22}}
\end{array}\right),
$$

is Markov.

Proof By the proposition, we have that the measure $\mu$ reduces to the Markov measure for matrices.

Lemma 2. For $V_{i}$ linear maps and $\rho_{0}$ fixed point for $\Lambda=\sum_{i} V_{i} \rho V_{i}^{*}$, we have for any $m, n$,

$$
\begin{gathered}
\mu\left(X_{1}=x_{1}, X_{2}=x_{2}, \ldots, X_{n}=x_{n}\right)= \\
\mu\left(X_{m}=x_{1}, X_{m+1}=x_{2}, \ldots, X_{m+n}=x_{n}\right)
\end{gathered}
$$

Proof We prove the lemma for the case in which we have two possible states 1 and 2 . We have

$$
\begin{gathered}
\mu\left(X_{m}=x_{1}, X_{m+1}=x_{2}, \ldots, X_{m+n}=x_{n}\right) \\
=\sum_{i_{1}, \ldots, i_{m-1}} \mu\left(X_{1}=i_{1}, X_{2}=i_{2}, \ldots, X_{m-1}=i_{m-1}, X_{m}=x_{1}, \ldots, X_{m+n}=x_{n}\right) \\
=\sum_{i_{2}, \ldots, i_{m-1}} \operatorname{tr}\left(V_{x_{n}} \cdots V_{x_{1}} V_{i_{m-1}} \cdots V_{i_{2}} V_{1} \rho_{0} V_{1}^{*} V_{i_{2}}^{*} \cdots\right) \\
+\operatorname{tr}\left(V_{x_{n}} \cdots V_{x_{1}} V_{i_{m-1}} \cdots V_{i_{2}} V_{2} \rho_{0} V_{2}^{*} V_{i_{2}}^{*} \cdots\right) \\
=\sum_{i_{2}, \ldots, i_{m-1}} \operatorname{tr}\left(V_{x_{n}} \cdots V_{x_{1}} V_{i_{m-1}} \cdots V_{i_{2}} \rho_{0} V_{i_{2}}^{*} V_{i_{3}}^{*} \cdots V_{i_{m-1}}^{*} V_{x_{1}}^{*} \cdots V_{x_{n}}^{*}\right)
\end{gathered}
$$

Repeating the procedure above for $i_{2}, i_{3}$, etc. we get

$$
\mu\left(X_{m}=x_{1}, X_{m+1}=x_{2}, \ldots, X_{m+n}=x_{n}\right)=\operatorname{tr}\left(V_{x_{n}} \cdots V_{x_{1}} \rho_{0} V_{x_{1}}^{*} \cdots V_{x_{n}}^{*}\right)
$$

This concludes the proof. 
Example 10. Let us make an inspection with respect to the ChapmanKolmogorov equation, that is, we would like to know if the equality

$$
\mu_{i j}(m+n)=\sum_{k} \mu_{i k}(m) \mu_{k j}(n)
$$

holds, where

$$
\mu_{i j}(n)=\mu\left(X_{m+n}=j \mid X_{m}=i\right)
$$

Take for instance, $m=n=i=j=1$. Then

$$
\begin{aligned}
& \sum_{k} \mu_{i k}(m) \mu_{k j}(n)=\mu_{11}(1) \mu_{11}(1)+\mu_{12}(1) \mu_{21}(1) \\
= & \frac{\operatorname{tr}\left(V_{1} V_{1} \rho V_{1}^{*} V_{1}^{*}\right)^{2}}{\operatorname{tr}\left(V_{1} \rho V_{1}^{*}\right)^{2}}+\frac{\operatorname{tr}\left(V_{2} V_{1} \rho V_{1}^{*} V_{2}^{*}\right)}{\operatorname{tr}\left(V_{1} \rho V_{1}^{*}\right)} \frac{\operatorname{tr}\left(V_{1} V_{2} \rho V_{2}^{*} V_{1}^{*}\right)}{\operatorname{tr}\left(V_{2} \rho V_{2}^{*}\right)}
\end{aligned}
$$

and

$$
\begin{gathered}
\mu_{i j}(m+n)=\mu_{11}(2)=\mu\left(X_{3}=1 \mid X_{1}=1\right) \\
=\frac{\operatorname{tr}\left(V_{1} V_{1} V_{1} \rho V_{1}^{*} V_{1}^{*} V_{1}^{*}\right)}{\operatorname{tr}\left(V_{1} \rho V_{1}^{*}\right)}+\frac{\operatorname{tr}\left(V_{1} V_{2} V_{1} \rho V_{1}^{*} V_{2}^{*} V_{1}^{*}\right)}{\operatorname{tr}\left(V_{1} \rho V_{1}^{*}\right)}
\end{gathered}
$$

Now let $V_{1}, V_{2}$ be given by (52), then we obtain classic calculations, so the Chapman-Kolmogorov equation holds. Now take

$$
V_{1}=\left(\begin{array}{ll}
1 & 0 \\
0 & 0
\end{array}\right), \quad V_{2}=\left(\begin{array}{ll}
1 & 0 \\
0 & 2
\end{array}\right)
$$

then we get, from (66) and (67):

$$
\frac{\operatorname{tr}\left(V_{1} V_{1} \rho V_{1}^{*} V_{1}^{*}\right)^{2}}{\operatorname{tr}\left(V_{1} \rho V_{1}^{*}\right)^{2}}+\frac{\operatorname{tr}\left(V_{2} V_{1} \rho V_{1}^{*} V_{2}^{*}\right)}{\operatorname{tr}\left(V_{1} \rho V_{1}^{*}\right)} \frac{\operatorname{tr}\left(V_{1} V_{2} \rho V_{2}^{*} V_{1}^{*}\right)}{\operatorname{tr}\left(V_{2} \rho V_{2}^{*}\right)}=1+\frac{\rho_{11}}{\rho_{11}+4 \rho_{22}}
$$

and

$$
\frac{\operatorname{tr}\left(V_{1} V_{1} V_{1} \rho V_{1}^{*} V_{1}^{*} V_{1}^{*}\right)}{\operatorname{tr}\left(V_{1} \rho V_{1}^{*}\right)}+\frac{\operatorname{tr}\left(V_{1} V_{2} V_{1} \rho V_{1}^{*} V_{2}^{*} V_{1}^{*}\right)}{\operatorname{tr}\left(V_{1} \rho V_{1}^{*}\right)}=1+1=2
$$

Then in this case we have that the Chapman-Kolmogorov equation holds if and only if $\rho_{22}=0$ that is, if $\rho_{11}=1$. Also, we note that $\sum_{i} V_{i}^{*} V_{i} \neq I$. To conclude this example, we take $V_{1}$ and $V_{2}$ with $\sum_{i} V_{i}^{*} V_{i}=I$, namely,

$$
V_{1}=\left(\begin{array}{cc}
\frac{1}{\sqrt{3}} & 0 \\
0 & 0
\end{array}\right), \quad V_{2}=\left(\begin{array}{cc}
\sqrt{\frac{2}{3}} & 0 \\
0 & 1
\end{array}\right)
$$

Take for instance $\rho_{0}=\frac{1}{4}|1\rangle\left\langle 1\left|+\frac{3}{4}\right| 2\right\rangle\langle 2|$, a fixed point for the associated $\Lambda$. A simple calculation shows that (66) and (67) are different. Therefore our calculation shows that the Chapman-Kolmogorov equation does not hold in general (for our setting). 
We would like to obtain a nonhomogeneous version for the measure we defined in (37) in the homogeneous case, i.e., we are looking for a measure induced by a nonhomogeneous QIFS. Let $W_{i}, i=1, \ldots, k$ be linear operators such that $\sum_{i} W_{i}^{*} W_{i}=I$. Let $\rho_{0} \in \mathcal{M}_{N}$. Define

$$
\begin{gathered}
\mu\left(X_{1}=x_{1}, \ldots, X_{n}=x_{n}\right):= \\
=\operatorname{tr}\left(W_{x_{1}} \rho_{0} W_{x_{1}}^{*}\right) \frac{\operatorname{tr}\left(W_{x_{2}} V_{x_{1}} \rho_{0} V_{x_{1}}^{*} W_{x_{2}}^{*}\right)}{\operatorname{tr}\left(V_{x_{1}} \rho_{0} V_{x_{1}}^{*}\right)} \frac{\operatorname{tr}\left(W_{x_{3}} V_{x_{2}} V_{x_{1}} \rho_{0} V_{x_{1}}^{*} V_{x_{2}}^{*} W_{x_{3}}^{*}\right)}{\operatorname{tr}\left(V_{x_{2}} V_{x_{1}} \rho_{0} V_{x_{1}}^{*} V_{x_{2}}^{*}\right)} \times \cdots \\
\cdots \times \frac{\operatorname{tr}\left(W_{x_{n-1}} V_{x_{n-2}} \cdots V_{x_{1}} \rho_{0} V_{x_{1}}^{*} \cdots V_{x_{n-2}}^{*} W_{x_{n-1}}^{*}\right)}{\operatorname{tr}\left(V_{x_{n-2}} \cdots V_{x_{1}} \rho_{0} V_{x_{1}}^{*} \cdots V_{x_{n-2}}^{*}\right)} \times \\
\times \frac{\operatorname{tr}\left(W_{x_{n}} V_{x_{n-1}} \cdots V_{x_{1}} \rho_{0} V_{x_{1}}^{*} \cdots V_{x_{n-1}}^{*} W_{x_{n}}^{*}\right)}{\operatorname{tr}\left(V_{x_{n-1}} \cdots V_{x_{1}} \rho_{0} V_{x_{1}}^{*} \cdots V_{x_{n-1}}^{*}\right)}
\end{gathered}
$$

that is,

$$
\begin{gathered}
\mu\left(X_{1}=x_{1}, \ldots, X_{n}=x_{n}\right):= \\
\operatorname{tr}\left(W_{x_{1}} \rho_{0} W_{x_{1}}^{*}\right) \prod_{i=2}^{n} \frac{\operatorname{tr}\left(W_{x_{i}} V_{x_{i-1}} \cdots V_{x_{1}} \rho_{0} V_{x_{1}}^{*} \cdots V_{x_{i-1}}^{*} W_{x_{i}}^{*}\right)}{\operatorname{tr}\left(V_{x_{i-1}} V_{x_{i-2}} \cdots V_{x_{1}} \rho_{0} V_{x_{1}}^{*} \cdots V_{x_{i-2}}^{*} V_{x_{i-1}}^{*}\right)}
\end{gathered}
$$

Remark A calculation shows that if we suppose $\sum_{i} W_{i}^{*} W_{i}=I$, then

$$
\sum_{i_{1}, \ldots i_{n}} \mu\left(\overline{i_{1} \cdots i_{n}}\right)=1
$$

Besides, if we suppose that $W_{i}=V_{i}$ for all $i$, then we recover the measure definition for homogeneous QSP.

Consider a QIFS $\mathcal{F}=\left\{\mathcal{M}_{N}, F_{i}, p_{i}\right\}_{i=1, \ldots, k}$, where

$$
F_{i}(\rho)=\frac{V_{i} \rho V_{i}^{*}}{\operatorname{tr}\left(V_{i} \rho V_{i}^{*}\right)}
$$

where the $V_{i}$ are linear and $p_{i}(\rho)=\operatorname{tr}\left(W_{i} \rho W_{i}^{*}\right), \operatorname{com} \sum_{i} W_{i}^{*} W_{i}=I$.

Definition 18. We say that the pair $\left(\left\{X_{n}\right\}_{n \in \mathbb{N}}, \mu\right), X_{n}: \Omega \rightarrow\{1, \ldots, k\}$, is a Quantum Stochastic Process associated to the nonhomogeneous QIFS $\mathcal{F}$ if $\mu$ is defined by (73), where $\rho_{0} \in \mathcal{M}_{N}$ is any density operator.

Remark In the definition above we can, of course, consider the particular case in which $\rho_{0}$ is a fixed point for

$$
\Lambda(\rho)=\sum_{i=1}^{k} \operatorname{tr}\left(W_{i} \rho W_{i}^{*}\right) \frac{V_{i} \rho V_{i}^{*}}{\operatorname{tr}\left(V_{i} \rho V_{i}^{*}\right)},
$$


induced by the QIFS $\mathcal{F}$.

Recall that by lemma 2, a homogeneous QSP is always stationary. This is no longer true in general for nonhomogeneous QSP.

Example 11. Let $\left\{X_{n}\right\}_{n \in \mathbb{N}}$ be a QSP induced by a nonhomogeneous QIFS. We would like to know whether

$$
\mu\left(X_{1}=1, X_{2}=2\right)=\mu\left(X_{2}=1, X_{3}=2\right)
$$

By definition we have:

$$
\mu\left(X_{1}=1, X_{2}=2\right)=\operatorname{tr}\left(W_{1} \rho_{0} W_{1}^{*}\right) \frac{\operatorname{tr}\left(W_{2} V_{1} \rho_{0} V_{1}^{*} W_{2}^{*}\right)}{\operatorname{tr}\left(V_{1} \rho_{0} V_{1}^{*}\right)}
$$

And also

$$
\begin{aligned}
\mu\left(X_{2}=1\right. & \left.X_{3}=2\right)=\mu\left(X_{1}=1, X_{2}=1, X_{3}=2\right)+\mu\left(X_{1}=2, X_{2}=1, X_{3}=2\right) \\
= & \operatorname{tr}\left(W_{1} \rho_{0} W_{1}^{*}\right) \frac{\operatorname{tr}\left(W_{1} V_{1} \rho_{0} V_{1}^{*} W_{1}^{*}\right)}{\operatorname{tr}\left(V_{1} \rho_{0} V_{1}^{*}\right)} \frac{\operatorname{tr}\left(W_{2} V_{1} V_{1} \rho_{0} V_{1}^{*} V_{1}^{*} W_{2}^{*}\right)}{\operatorname{tr}\left(V_{1} V_{1} \rho_{0} V_{1}^{*} V_{1}^{*}\right)} \\
& +\operatorname{tr}\left(W_{2} \rho_{0} W_{2}^{*}\right) \frac{\operatorname{tr}\left(W_{1} V_{2} \rho_{0} V_{2}^{*} W_{1}^{*}\right)}{\operatorname{tr}\left(V_{2} \rho_{0} V_{2}^{*}\right)} \frac{\operatorname{tr}\left(W_{2} V_{1} V_{2} \rho_{0} V_{2}^{*} V_{1}^{*} W_{2}^{*}\right)}{\operatorname{tr}\left(V_{1} V_{2} \rho_{0} V_{2}^{*} V_{1}^{*}\right)} \\
= & \operatorname{tr}\left[W _ { 2 } V _ { 1 } \left[\operatorname{tr}\left(W_{1} \rho_{0} W_{1}^{*}\right) \frac{V_{1} \rho_{0} V_{1}^{*}}{\operatorname{tr}\left(V_{1} \rho_{0} V_{1}^{*}\right)}\left(\frac{\operatorname{tr}\left(W_{1} V_{1} \rho_{0} V_{1}^{*} W_{1}^{*}\right)}{\operatorname{tr}\left(V_{1} V_{1} \rho_{0} V_{1}^{*} V_{1}^{*}\right)}\right)+\right.\right. \\
& \left.\left.+\operatorname{tr}\left(W_{2} \rho_{0} W_{2}^{*}\right) \frac{V_{2} \rho_{0} V_{2}^{*}}{\operatorname{tr}\left(V_{2} \rho_{0} V_{2}^{*}\right)}\left(\frac{\operatorname{tr}\left(W_{1} V_{2} \rho_{0} V_{2}^{*} W_{1}^{*}\right)}{\operatorname{tr}\left(V_{1} V_{2} \rho_{0} V_{2}^{*} V_{1}^{*}\right)}\right)\right] V_{1}^{*} W_{2}^{*}\right]
\end{aligned}
$$

Note that in the homogeneous case we have that both fractions in parenthesis on equation (77) are equal to 1 , so if $\rho_{0}$ is a fixed point for $\Lambda$, then we have stationarity, a fact we have already proved. But in the nonhomogeneous case, the terms in parenthesis are not equal to 1 in general.

\section{A definition of entropy for QIFS}

We will present a notion of entropy for "invariant" (or "stationary") measures with support on density matrices. This definition is obtained by adapting the reasoning described in [5], [12] and [13] to the present situation. The main idea is to define this concept via the Ruelle operator and to avoid the use of partitions. 
Denote by $p$ an arbitrary choice of mappings $p_{i}: \mathcal{M}_{N} \rightarrow \mathbb{R}, i=1, \ldots, k$ for a certain $k$. Let

$$
m_{b}\left(\mathcal{M}_{N}\right):=\left\{f: \mathcal{M}_{N} \rightarrow \mathbb{R}: \mathrm{f} \text { is measurable and bounded }\right\}
$$

Let $\mathcal{U}_{p}: m_{b}\left(\mathcal{M}_{N}\right) \rightarrow m_{b}\left(\mathcal{M}_{N}\right)$,

$$
\left(\mathcal{U}_{p} f\right)(\rho):=\sum_{i=1}^{k} p_{i}(\rho) f\left(F_{i}(\rho)\right)
$$

Let us consider all possible choices of mappings $p_{i}: \mathcal{M}_{N} \rightarrow \mathbb{R}$ which satisfy

$$
\mathcal{U}_{p} 1=1
$$

Each $p$ determines an operator $\mathcal{U}_{p}$. The set of all possible $p$ that satisfy (78) will be denoted by $P$.

Let $\left(\mathcal{M}_{N}, F_{i}, p_{i}\right)_{i=1, \ldots k}$ be a QIFS. An example of Markov operator for measures is the one we defined before, given by $\mathcal{V}_{p}: M^{1}\left(\mathcal{M}_{N}\right) \rightarrow M^{1}\left(\mathcal{M}_{N}\right)$,

$$
\left(\mathcal{V}_{p} \nu\right)(B)=\sum_{i=1}^{k} \int_{F_{i}^{-1}(B)} p_{i} d \nu,
$$

which we will call the Markov operator Markov induced by the $p_{i}$. That is, we will consider all $\mathcal{V}_{p}$ with $p \in P$. We say that $\nu$ is invariant for the $F_{i}$ if for some $p \in P$ we have that $\mathcal{V}_{p} \nu=\nu$.

Let $\mathcal{M}_{F}$ be the set of all invariant measures for a fixed choice of the dynamics $F_{i}, i=1, \ldots, k$. For such measures $\nu \in \mathcal{M}_{F}$, and based on [5], [12] and [13], define

$$
h_{0}(\nu):=\inf _{f \in \mathbb{B}^{+}} \int \log \left(\sum_{i=1}^{k} \frac{f \circ F_{i}}{f}\right) d \nu
$$

Above, $\mathbb{B}^{+}$denotes the bounded, positive, borelean functions on $\mathcal{M}_{N}$.

Proposition 4. For $\nu \in \mathcal{M}_{F}$, we have that $0 \leq h_{0}(\nu) \leq \log k$.

In order to prove this proposition, we need the following lemma.

Lemma 3. [13] Let $\beta \geq 1+\alpha$ and numbers $a_{i} \in[1+\alpha, \beta], i=1, \ldots, k$. Then there exists $\epsilon \geq 1$ such that

$$
\log \left(\epsilon \sum_{i=1}^{k} a_{i}\right) \geq \sum_{i=1}^{k} \log \left(\epsilon a_{i}\right) .
$$


The proof of this lemma follows by choosing

$$
\epsilon=\exp \left(\frac{1}{k} \frac{\log \sum_{i=1}^{k} a_{i}}{\sum_{i=1}^{k} \log a_{i}}\right)
$$

Lemma 4. If $f \in \mathbb{B}^{+}$and $\nu \in \mathcal{M}_{F}$ then

$$
\sum_{i=1}^{k} \int f \circ F_{i} d \nu \geq \int f d \nu
$$

Proof First suppose that $f=1_{B}$, where $B$ is a measurable set. We have that

$$
\begin{gathered}
\sum_{i=1}^{k} \int 1_{B} \circ F_{i} d \nu \geq \sum_{i=1}^{k} \int p_{i}(x) 1_{B}\left(F_{i}(x)\right) d \nu(x)=\sum_{i=1}^{k} \int_{F_{i}^{-1}(B)} p_{i}(x) d \nu(x) \\
=\mathcal{V}_{p}(\nu)(B)=\nu(B)=\int 1_{B} d \nu
\end{gathered}
$$

Then, assume that $f=\sum_{j=1}^{l} b_{j} 1_{B_{j}}$, i.e., a simple function. Then

$$
\begin{gathered}
\sum_{i=1}^{k} \int \sum_{j=1}^{l} b_{j} 1_{B_{j}} \circ F_{i} d \nu=\sum_{j=1}^{l} b_{j} \sum_{i=1}^{k} \int 1_{B_{j}} \circ F_{i} d \nu \\
\geq \sum_{j=1}^{l} b_{j} \sum_{i=1}^{k} \int p_{i}(x) 1_{B_{j}}\left(F_{i}(x)\right) d \nu=\sum_{j=1}^{l} b_{j} \mathcal{V}_{p}(\nu)\left(B_{j}\right) \\
=\sum_{j=1}^{l} b_{j} \nu\left(B_{j}\right)=\int f d \nu
\end{gathered}
$$

Now let $f=\lim _{n} f_{n}$, a limit of a sequence of simple functions. Note that we suppose $f \in \mathbb{B}^{+}$, so $f$ is bounded, and since $\nu$ is a probability measure on $\mathcal{M}_{N}$, it follows that $f$ is integrable. By the bounded convergence theorem, we have that

$$
\begin{gathered}
\sum_{i=1}^{k} \int f \circ F_{i} d \nu=\sum_{i=1}^{k} \int \lim _{n} f_{n} \circ F_{i} d \nu=\lim _{n} \sum_{i=1}^{k} \int f_{n} \circ F_{i} d \nu \\
\geq \lim _{n} \int f_{n} d \nu=\int \lim _{n} f_{n} d \nu=\int f d \nu
\end{gathered}
$$


The following proof is an adaptation of results seen in [13].

Proof of proposition 4 Let us restrict the proof for the case in which we have a QIFS $\left(\mathcal{M}_{N}, F_{i}, p_{i}\right)_{i=1, \ldots, k}$, where $F_{i}(\rho)=V_{i} \rho V_{i}^{*}$, with linear $V_{i}$.

First note that if $f \equiv 1$, we have $\int \log \left(\sum_{i=1}^{k} 1\right) d \nu=\log k$, so $h_{0}(\nu) \leq$ $\log k$.

Let $I=\int \log \left(\sum_{i=1}^{k} \frac{f \circ F_{i}}{f}\right) d \nu$ and suppose, without loss of generality, that $1+\alpha \leq f \leq \beta$ (note that this integral is invariant by the projective mapping $f \rightarrow \lambda f)$. Then

$$
I=\int \log \left(\sum_{i=1}^{k} \frac{\epsilon f \circ F_{i}}{\epsilon f}\right) d \nu=\int \log \left(\sum_{i=1}^{k} \epsilon f \circ F_{i}\right) d \nu-\int \log (\epsilon f) d \nu
$$

Define

$$
a_{i}=f \circ F_{i}(\rho)
$$

Then

$$
\epsilon(\rho)=\exp \left(\frac{1}{k} \frac{\log \sum_{i=1}^{k} f \circ F_{i}}{\sum_{i=1}^{k} \log f \circ F_{i}}\right) \geq \epsilon_{0} \geq 1,
$$

by the compactness of $\mathcal{M}_{N}$. With such choice we obtain, by lemma (3),

$$
\log \left(\epsilon_{0} \sum_{i=1}^{k} f \circ F_{i}\right) \geq \sum_{i=1}^{k} \log \left(\epsilon_{0} f \circ F_{i}\right)
$$

Apply (80) on (79), then

$$
I \geq \sum_{i=1}^{k} \int \log \left(\epsilon_{0} f \circ F_{i}\right) d \nu-\int \log \left(\epsilon_{0} f\right) d \nu
$$

Then by lemma (4) applied on the function $\log (\epsilon f)$ (note that we have $\log \left(\epsilon_{0} f\right) \in \mathbb{B}^{+}$because $\left.\epsilon_{0} \geq 1\right)$, we get

$$
I \geq \int \log (\epsilon f) d \nu-\int \log (\epsilon f) d \nu=0
$$

The computation in the next example shows that the concept of entropy described here is different from the one presented in [1] [2].

Example 12. We will consider an example of a probability $\eta$ such that $\mathcal{V}(\eta)=\eta$ and we will compute the entropy of $\eta$. 
Suppose a QIFS, such that

$$
p_{i}(\rho)=\operatorname{tr}\left(W_{i} \rho W_{i}^{*}\right), \quad \sum_{i} W_{i}^{*} W_{i}=I, \quad F_{i}(\rho)=\frac{V_{i} \rho V_{i}^{*}}{\operatorname{tr}\left(V_{i} \rho V_{i}^{*}\right)}
$$

for $i=1, \ldots, k$. Denote $m_{b}\left(\mathcal{M}_{N}\right)$ the space of bounded and measurable functions in $\mathcal{M}_{N}$.

Consider $\Lambda: \mathcal{M}_{N} \rightarrow \mathcal{M}_{N}$,

$$
\Lambda(\rho)=\sum_{i} p_{i}(\rho) F_{i}(\rho)=\sum_{i} \operatorname{tr}\left(W_{i} \rho W_{i}^{*}\right) \frac{V_{i} \rho V_{i}^{*}}{\operatorname{tr}\left(V_{i} \rho V_{i}^{*}\right)}
$$

Suppose there exists a density matrix $\rho$ which $\Lambda$-invariant. As we know, such state is the barycenter of $\mu$ which is $\mathcal{V}$-invariant [1].

Suppose $\mathcal{V} \mu=\mu$, then we can write

$$
\begin{gathered}
\int f d \mu=\int f d \mathcal{V} \mu=\sum_{i=1}^{k} \int p_{i}(\rho) f\left(F_{i}(\rho)\right) d \mu(\rho)= \\
\sum_{i} \int p_{i}(\rho) f\left(\frac{V_{i} \rho V_{i}^{*}}{\operatorname{tr}\left(V_{i} \rho V_{i}^{*}\right)}\right) d \mu=\sum_{i} \int \operatorname{tr}\left(W_{i} \rho W_{i}^{*}\right) f\left(\frac{V_{i} \rho V_{i}^{*}}{\operatorname{tr}\left(V_{i} \rho V_{i}^{*}\right)}\right) d \mu
\end{gathered}
$$

Therefore, for any $f \in m_{b}\left(\mathcal{M}_{N}\right)$, we got the condition

$$
\int f d \mu=\sum_{i} \int \operatorname{tr}\left(W_{i} \rho W_{i}^{*}\right) f\left(\frac{V_{i} \rho V_{i}^{*}}{\operatorname{tr}\left(V_{i} \rho V_{i}^{*}\right)}\right) d \mu
$$

Let us consider a particular example where $N=2, k=4$, and

$$
\begin{aligned}
& V_{1}=\left(\begin{array}{cc}
\sqrt{p_{11}} & 0 \\
0 & 0
\end{array}\right), V_{2}=\left(\begin{array}{cc}
0 & \sqrt{p_{12}} \\
0 & 0
\end{array}\right), \\
& V_{3}=\left(\begin{array}{cc}
0 & 0 \\
\sqrt{p_{21}} & 0
\end{array}\right), V_{4}=\left(\begin{array}{cc}
0 & 0 \\
0 & \sqrt{p_{22}}
\end{array}\right),
\end{aligned}
$$

in such way that the $p_{i j}$ are the entries of a column stochastic matrix $P$. Let $\pi=\left(\pi_{1}, \pi_{2}\right)$ be a vector such that $P \pi=\pi$. A simple calculation shows that for $\rho$, the density matrix such that has entries $\rho_{i j}$, we have

$$
\begin{aligned}
& V_{1} \rho V_{1}^{*}=\left(\begin{array}{cc}
p_{11} \rho_{11} & 0 \\
0 & 0
\end{array}\right), V_{2} \rho V_{2}^{*}=\left(\begin{array}{cc}
p_{12} \rho_{22} & 0 \\
0 & 0
\end{array}\right) \\
& V_{3} \rho V_{3}^{*}=\left(\begin{array}{cc}
0 & 0 \\
0 & p_{21} \rho_{11}
\end{array}\right), V_{4} \rho V_{4}^{*}=\left(\begin{array}{cc}
0 & 0 \\
0 & p_{22} \rho_{22}
\end{array}\right),
\end{aligned}
$$


and therefore

$$
\begin{aligned}
& \frac{V_{1} \rho V_{1}^{*}}{\operatorname{tr}\left(V_{1} \rho V_{1}^{*}\right)}=\left(\begin{array}{ll}
1 & 0 \\
0 & 0
\end{array}\right), \frac{V_{2} \rho V_{2}^{*}}{\operatorname{tr}\left(V_{2} \rho V_{2}^{*}\right)}=\left(\begin{array}{ll}
1 & 0 \\
0 & 0
\end{array}\right) \\
& \frac{V_{3} \rho V_{3}^{*}}{\operatorname{tr}\left(V_{3} \rho V_{3}^{*}\right)}=\left(\begin{array}{ll}
0 & 0 \\
0 & 1
\end{array}\right), \frac{V_{4} \rho V_{4}^{*}}{\operatorname{tr}\left(V_{4} \rho V_{4}^{*}\right)}=\left(\begin{array}{ll}
0 & 0 \\
0 & 1
\end{array}\right)
\end{aligned}
$$

that is, the above values do not depend on $\rho$.

Define

$$
\rho_{x}=\left(\begin{array}{ll}
1 & 0 \\
0 & 0
\end{array}\right), \rho_{y}=\left(\begin{array}{ll}
0 & 0 \\
0 & 1
\end{array}\right)
$$

and

$$
\eta=\pi_{1} \delta_{\rho_{x}}+\pi_{2} \delta_{\rho_{y}}
$$

Note that the barycenter of $\eta$ is

$$
\rho_{\eta}=\pi_{1} \rho_{x}+\pi_{2} \rho_{y}=\pi_{1}\left(\begin{array}{ll}
1 & 0 \\
0 & 0
\end{array}\right)+\pi_{2}\left(\begin{array}{cc}
0 & 0 \\
0 & 1
\end{array}\right)=\left(\begin{array}{cc}
\pi_{1} & 0 \\
0 & \pi_{2}
\end{array}\right)
$$

One can show directly that $\mathcal{V}(\eta)=\eta$ (see [1]). Define

$$
\rho_{1}=\left(\begin{array}{ll}
1 & 0 \\
0 & 0
\end{array}\right), \rho_{2}=\left(\begin{array}{ll}
0 & 0 \\
0 & 1
\end{array}\right)
$$

and also

$$
\eta=\pi_{1} \delta_{\rho_{1}}+\pi_{2} \delta_{\rho_{2}}
$$

Note that the barycenter of $\eta$ is

$$
\rho_{\eta}=\pi_{1} \rho_{1}+\pi_{2} \rho_{2}=\pi_{1}\left(\begin{array}{ll}
1 & 0 \\
0 & 0
\end{array}\right)+\pi_{2}\left(\begin{array}{cc}
0 & 0 \\
0 & 1
\end{array}\right)=\left(\begin{array}{cc}
\pi_{1} & 0 \\
0 & \pi_{2}
\end{array}\right)
$$

From this it will also follow that $\mathcal{V} \eta=\eta[1]$. We will show that the entropy of such $\eta$ is $\log (2)-\pi_{1} \log \left(\pi_{1}\right)-\pi_{2} \log \left(\pi_{2}\right)$. Remember that

$$
\begin{gathered}
\int \log \left(\sum_{i} \frac{f \circ F_{i}}{f}\right) d \mu \\
=\int \log \left(\sum_{i} f\left(\frac{V_{i} \rho V_{i}^{*}}{\operatorname{tr}\left(V_{i} \rho V_{i}^{*}\right)}\right)\right) d \mu-\sum_{i} \int \operatorname{tr}\left(W_{i} \rho W_{i}^{*}\right) \log f\left(\frac{V_{i} \rho V_{i}^{*}}{\operatorname{tr}\left(V_{i} \rho V_{i}^{*}\right)}\right) d \mu .
\end{gathered}
$$

For such choice of $V_{i}$ take

$$
c_{i}=f\left(\frac{V_{i} \rho V_{i}^{*}}{\operatorname{tr}\left(V_{i} \rho V_{i}^{*}\right)}\right), \quad i=1, \ldots, 4
$$

Note that

$$
c_{1}=c_{2}, \quad c_{3}=c_{4}
$$


Then we can write

$$
\int \log \left(\sum_{i} \frac{f \circ F_{i}}{f}\right) d \eta=\int \log \left(\sum_{i} c_{i}\right) d \eta-\sum_{i} \int \operatorname{tr}\left(W_{i} \rho W_{i}^{*}\right) \log c_{i} d \eta \text {. }
$$

Therefore

$$
\begin{gathered}
\int \log \left(\sum_{i} \frac{f \circ F_{i}}{f}\right) d \eta=\pi_{1} \log \left(\sum_{i} c_{i}\right)+\pi_{2} \log \left(\sum_{i} c_{i}\right) \\
-\sum_{i}\left[\operatorname{tr}\left(V_{i} \rho_{1} V_{i}^{*}\right) \pi_{1} \log \left(c_{i}\right)+\operatorname{tr}\left(V_{i} \rho_{2} V_{i}^{*}\right) \pi_{2} \log \left(c_{i}\right)\right] \\
=\pi_{1} \log \left(2 c_{1}+2 c_{3}\right)+\pi_{2} \log \left(2 c_{1}+2 c_{3}\right) \\
-\sum_{i}\left[\operatorname{tr}\left(V_{i} \rho_{1} V_{i}^{*}\right) \pi_{1} \log \left(c_{i}\right)+\operatorname{tr}\left(V_{i} \rho_{2} V_{i}^{*}\right) \pi_{2} \log \left(c_{i}\right)\right] \\
=\log \left(2\left(c_{1}+c_{3}\right)\right)-\sum_{i}\left[\operatorname{tr}\left(V_{i} \rho_{1} V_{i}^{*}\right) \pi_{1} \log \left(c_{i}\right)+\operatorname{tr}\left(V_{i} \rho_{2} V_{i}^{*}\right) \pi_{2} \log \left(c_{i}\right)\right] \\
=\log \left(2\left(c_{1}+c_{3}\right)\right) \\
-\left[\pi_{1}\left(p_{11}^{2} \log \left(c_{1}\right)+p_{12} p_{21} \log \left(c_{2}\right)+p_{21} p_{11} \log \left(c_{3}\right)+p_{22} p_{21} \log \left(c_{4}\right)\right)\right. \\
\left.+\pi_{2}\left(p_{11} p_{12} \log \left(c_{1}\right)+p_{12} p_{22} \log \left(c_{2}\right)+p_{21} p_{12} \log \left(c_{3}\right)+p_{22}^{2} \log \left(c_{4}\right)\right)\right] \\
=\log \left(2\left(c_{1}+c_{3}\right)\right) \\
-\left[p_{11} \log \left(c_{1}\right)\left(\pi_{1} p_{11}+\pi_{2} p_{12}\right)+p_{12} \log \left(c_{2}\right)\left(\pi_{1} p_{21}+\pi_{2} p_{22}\right)\right. \\
\left.+p_{21} \log \left(c_{3}\right)\left(\pi_{1} p_{11}+\pi_{2} p_{12}\right)+p_{22} \log \left(c_{4}\right)\left(\pi_{1} p_{21}+\pi_{2} p_{22}\right)\right] \\
=\log \left(2\left(c_{1}+c_{3}\right)\right) \\
-\left[\pi_{1} p_{11} \log \left(c_{1}\right)+\pi_{2} p_{12} \log \left(c_{2}\right)+\pi_{1} p_{21} \log \left(c_{3}\right)+\pi_{2} p_{22} \log \left(c_{4}\right)\right] \\
=\log \left(2\left(c_{1}+c_{3}\right)\right)-\left(\pi_{1} \log \left(c_{1}\right)+\pi_{2} \log \left(c_{3}\right)\right)
\end{gathered}
$$

Finally,

$$
\int \log \left(\sum_{i} \frac{f \circ F_{i}}{f}\right) d \eta=\log \left(2\left(c_{1}+c_{3}\right)\right)-\left(\pi_{1} \log \left(c_{1}\right)+\pi_{2} \log \left(c_{3}\right)\right) .
$$

Now we will use Lagrange multipliers. Define $b: \mathbb{R}_{+}^{2} \rightarrow \mathbb{R}$, where $\mathbb{R}_{+}^{2}$ is the set of positive coordinates, by

$$
b(x, y)=\log (2(x+y))-\left(\pi_{1} \log (x)+\pi_{2} \log (y)\right)
$$


We impose the restriction

$$
x+y=a
$$

for fixed $a>0$. We will get bellow the critical point of $b$ under such restriction. After that we consider a general $a>0$.

Define

and

$$
g(x, y)=x+y-a
$$

Then, $\nabla \Gamma=0$ implies

$$
\Gamma(x, y, \lambda)=b+\lambda g
$$

$$
\begin{gathered}
\frac{1}{x+y}-\frac{\pi_{1}}{x}+\lambda=0 \\
\frac{1}{x+y}-\frac{\pi_{2}}{y}+\lambda=0 \\
x+y=a
\end{gathered}
$$

from which follows

$$
x=\pi_{1} a, \quad y=\pi_{2} a .
$$

Therefore,

From (94) we get

$$
c_{1}=c_{2}=\pi_{1} a, \quad c_{3}=c_{4}=\pi_{2} a
$$

$$
\begin{gathered}
\int \log \left(\sum_{i} \frac{f \circ F_{i}}{f}\right) d \eta=\log \left(2\left(\pi_{1} a+\pi_{2} a\right)\right)-\left(\pi_{1} \log \left(\pi_{1} a\right)+\pi_{2} \log \left(\pi_{2} a\right)\right) \\
=\log (2 a)-\pi_{1} \log \left(\pi_{1} a\right)-\pi_{2} \log \left(\pi_{2} a\right) \\
=\log (2)+\log (a)-\pi_{1} \log \left(\pi_{1}\right)-\pi_{1} \log (a)-\pi_{2} \log \left(\pi_{2}\right)-\pi_{2} \log (a) \\
=\log (2)-\pi_{1} \log \left(\pi_{1}\right)-\pi_{2} \log \left(\pi_{2}\right)
\end{gathered}
$$

This value of entropy is different from the value computed in the same example of QIFS in [1], [2] which is $-\sum_{i, j} \pi_{i} p_{j i} \log p_{j i}$ (Example 7 in section $11[1])$.

Given the expression

$$
h_{0}(\nu):=\inf _{f \in \mathbb{B}^{+}} \int \log \left(\sum_{i=1}^{k} \frac{f \circ F_{i}}{f}\right) d \nu,
$$

for a fixed probability $\nu$, which is invariant by the shift acting on the space $\Omega$, a natural question is to identify the $f$ which realizes the infimum above.

We will describe below the analysis of the classical case (in the sense of Stochastic Processes, and not QSP). Our purpose is to explain why 
the definition presented above is a natural generalization of the setting for Markov Processes. In the case the probability $\nu$ comes from a Markov Process this will be now derived.

Let $\Omega=I_{m}^{\mathbb{N}}$, where $I_{m}=\{1, \ldots, m\}$, and let $\mathcal{C}=\left\{C_{\iota}: \iota \in \cup_{n \in \mathbb{N}} I_{m}^{n}\right\}$ the collection of cylinder sets in $\Omega$, where

$$
C_{\iota}:=\left\{\omega \in I_{k}^{\mathbb{N}}: w(j)=i_{j}, j=1, \ldots, r, \iota=\left(i_{1}, \ldots, i_{r}\right) \in I_{m}^{r}\right\}
$$

and denote by $\sigma(\mathcal{C})$ the $\sigma$-algebra generated by the cylinders in $\Omega$.

Let $(P, \pi)$ be a Markov chain, so that $P=\left(p_{i j}\right)$ is a matrix of order $n$, with $p_{i j} \geq 0, \sum_{j} p_{i j}=1$ (row stochastic), and $\pi=\left(\pi_{1}, \ldots, \pi_{n}\right)$ is the left eigenvector with eigenvalue 1 . So $\pi P=\pi$, that is, $\sum_{i} \pi_{i} p_{i j}=\pi_{j}$.

Associated to the matrix $P$ we have the following measure.

Definition 19. The Markov measure (associated to the chain $(P, \pi)$ ) of a cylinder is defined as

$$
\nu\left(C_{\iota}\right):=\pi_{i_{1}} p_{i_{1} i_{2}} p_{i_{2} i_{3}} \cdots p_{i_{r-1} i_{r}}
$$

We are interested in the following problem: find the infimum $f$ in

$$
h_{0}(\nu):=\inf _{f \in \mathbb{B}^{+}} \int \log \left(\sum_{i=1}^{k} \frac{f \circ F_{i}}{f}\right) d \nu
$$

for such $\nu$ defined above.

We use the notation $\overline{i j}$ to denote the cylinder set in $I_{m}^{\mathbb{N}}$ which consists of the set of sequences $\left(w_{1}, w_{2}, \ldots\right)$ such that $w_{1}=i$ and $w_{2}=j$. Denote by $1_{\overline{i j}}$ the indicator function of $\overline{i j}$. To simplify, suppose $m=2$ so the alphabet considered contains only two symbols, denoted by 1 and 2. Define the following function $f: I_{2}^{\mathbb{N}} \rightarrow \mathbb{R}^{+}$,

$$
f(x)=\sum_{i, j=1}^{2} a_{i j} 1_{\overline{i j}}(x)
$$

where $a_{i j} \in \mathbb{R}^{+}$. That is, $f$ is a simple function, constant on $\overline{i j}$. In this form, $\log f=\sum_{i, j} \log a_{i j} 1_{\overline{i j}}$. 
Let us suppose that $F_{i}: I_{m}^{\mathbb{N}} \rightarrow I_{m}^{\mathbb{N}}$ is the mapping $F_{i}\left(w_{1}, w_{2}, \ldots\right)=$ $\left(i, w_{1}, w_{2}, \ldots\right)$. If $\nu$ is a Markov measure, we have

$$
\int_{I_{2}^{\mathbb{N}}} \log f d \nu=\int_{I_{2}^{\mathbb{N}}} \sum_{i, j=1}^{2} \log \left(a_{i j}\right) 1_{i j} d \nu=\sum_{i, j=1}^{2} \log \left(a_{i j}\right) \nu(\overline{i j})=\sum_{i, j=1}^{2} \pi_{i} p_{i j} \log a_{i j}
$$

Also, we have for $w=(i, j, \ldots)$,

$$
f \circ F_{l}(w)=\sum_{i, j} a_{i j} 1_{\overline{i j}}\left(F_{l}(w)\right)=a_{l i}
$$

To see that, note that by the expression above we have a sum of terms such that $(i, j)=(l, i)$, therefore $a_{i j}=a_{l i}$.

Then

$$
\begin{gathered}
\int \log \left(\sum_{i=1}^{2} \frac{f \circ F_{i}}{f}\right) d \nu=\int \log \left(\sum_{l=1}^{2} f \circ F_{l}\right) d \nu-\int \log f d \nu \\
=\int \log \left(\sum_{l=1}^{2} f \circ F_{l}\right) d \nu-\sum_{i, j=1}^{2} \pi_{i} p_{i j} \log a_{i j}
\end{gathered}
$$

Note that for any $w \in I_{m}^{\mathbb{N}}, w=(1, \ldots)$ or $w=(2, \ldots)$. Then, by (105) we get

$$
\sum_{l=1}^{2} f \circ F_{l}(w)= \begin{cases}a_{11}+a_{21} & \text { se } w=(1, \ldots) \\ a_{12}+a_{22} & \text { se } w=(2, \ldots)\end{cases}
$$

Now fix $a_{i j}=p_{j i}$, where $p_{i j}$ are the entries of the row stochastic matrix $P$ initially fixed. Then we get $a_{11}+a_{21}=p_{11}+p_{12}=1$ e $a_{12}+a_{22}=$ $p_{21}+p_{22}=1$. Therefore for such choice of $a_{i j}$ and for any $w \in I_{m}^{\mathbb{N}}$, the sum (107) equals 1 . So, by (106), we get

$$
\int \log \left(\sum_{i=1}^{2} \frac{f \circ F_{i}}{f}\right) d \nu=-\sum_{i, j=1}^{2} \pi_{i} p_{i j} \log p_{i j}=H(P)
$$

Therefore,

$$
\inf _{f \in \mathbb{B}^{+}} \int \log \left(\sum_{i=1}^{2} \frac{f \circ F_{i}}{f}\right) d \nu \leq H(P)
$$


Now note that any positive function $f$ can be written as

$$
f(w)=\sum_{i, j=1}^{2} a_{i j} p_{j i} 1_{\overline{j i}}(w)
$$

Define

$$
u(w):=\sum_{i, j=1}^{2} a_{i j} 1 \overline{j i}(w)
$$

and

$$
g(w):=\sum_{i, j=1}^{2} p_{j i} 1_{\overline{j i}}(w)
$$

We have

$$
\begin{aligned}
& \int_{I_{2}^{\mathbb{N}}} \log f d \nu=\int_{I_{2}^{\mathbb{N}}} \sum_{i, j=1}^{2} \log \left(a_{i j} p_{j i}\right) 1_{\overline{j i}} d \nu=\sum_{i, j=1}^{2} \log \left(a_{i j} p_{j i}\right) \nu(\overline{j i}) \\
= & \sum_{i, j=1}^{2} \pi_{j} p_{j i} \log \left(a_{i j} p_{j i}\right)=\sum_{i, j=1}^{2} \pi_{j} p_{j i} \log \left(a_{i j}\right)+\sum_{i, j=1}^{2} \pi_{j} p_{j i} \log \left(p_{j i}\right)
\end{aligned}
$$

If $w=(i, j, \ldots)$, then $f \circ F_{l}(w)=a_{l i} p_{i l}$ and so

$$
\sum_{l} f \circ F_{l}=\sum_{l} a_{l i} p_{i l}
$$

We write

$$
\mathcal{L}_{g}(u)(w)=\sum_{l} f \circ F_{l}(w)=\sum_{l} \sum_{i, j} a_{i j} p_{j i} 1_{i j}\left(F_{l}(w)\right)
$$

We also have the following:

\section{Lemma 5.}

$$
\int \mathcal{L}_{g}(\log u) d \nu=\int \log u d \nu
$$

Proof We have

$$
\int \log u d \nu=\int \sum_{i, j} \log \left(a_{i j}\right) 1_{j i} d \nu=\sum_{i, j} \log \left(a_{i j}\right) \nu(\overline{j i})=\sum_{i, j} \log \left(a_{i j}\right) \pi_{j} p_{j i}
$$

And also

$$
\int \mathcal{L}_{g}(\log u) d \nu=\int \sum_{l} \sum_{i, j} \log \left(a_{i j}\right) p_{j i} 1_{\overline{i j}}\left(F_{l}(w)\right) d \nu
$$




$$
\begin{gathered}
=\sum_{i, j} \log \left(a_{i j}\right) p_{j i} \sum_{l} \int 1_{\overline{i j}}\left(F_{l}(w)\right) d \nu \\
=\sum_{i, j} \log \left(a_{i j}\right) p_{j i} \sum_{l} \nu(\overline{l j})=\sum_{i, j} \log \left(a_{i j}\right) p_{j i}\left(\pi_{1} p_{1 j}+\pi_{2} p_{2 j}\right)=\sum_{i, j} \log \left(a_{i j}\right) \pi_{j} p_{j i}
\end{gathered}
$$

So,

$$
\int \mathcal{L}_{g}(\log u) d \nu=\int \log u d \nu
$$

Then, by using (110), (112) and (113),

$$
\begin{gathered}
\int \log \left(\sum_{i=1}^{k} \frac{f \circ F_{i}}{f}\right) d \nu=\int \log \left(\sum_{l=1}^{2} f \circ F_{l}\right) d \nu-\int \log f d \nu \\
=\int \log \left(\sum_{l=1}^{2} f \circ F_{l}\right) d \nu-\left(\sum_{i, j=1}^{2} \pi_{j} p_{j i} \log \left(a_{i j}\right)+\sum_{i, j=1}^{2} \pi_{i} p_{i j} \log \left(p_{i j}\right)\right) \\
=\int \log \left(\mathcal{L}_{g}(u)\right) d \nu-\int \log u d \nu+H(P) \\
=\int \log \left(\mathcal{L}_{g}(u)\right) d \nu-\int \mathcal{L}_{g}(\log u) d \nu+H(P)
\end{gathered}
$$

We would like to show that

$$
\int \log \left(\mathcal{L}_{g}(u)\right) d \nu-\int \mathcal{L}_{g}(\log u) d \nu \geq 0
$$

This follows immediately if we show that for $w=(i, j, \ldots)$,

$$
\log \left(\mathcal{L}_{g}(u)\right)(w) \geq \mathcal{L}_{g}(\log u)(w)
$$

The last expression follows from convexity. Indeed, to prove the above inequality, it is enough to show that for any $w=(i, j, \ldots)$, we have

$$
\log \left(\sum_{l} a_{l i} p_{i l}\right) \geq \sum_{l} p_{i l} \log a_{l i}
$$

And such inequality is true, because the $p_{i l}$ are positive numbers with $\sum_{l} p_{i l}=1$, for any $i$, and the function $\log$ is concave.

Therefore we conclude from (117) and (118) that

$$
\int \log \left(\sum_{i=1}^{k} \frac{f \circ F_{i}}{f}\right) d \nu \geq H(P)
$$


Conclusion By (109) and (121) we conclude that if $\nu$ is a Markov measure associated to a stochastic matrix $P$, then

$$
\inf _{f \in \mathbb{B}^{+}} \int \log \left(\sum_{i=1}^{2} \frac{f \circ F_{i}}{f}\right) d \nu=H(P)
$$

and the function $f$ such that

$$
f(x)=\sum_{i, j=1}^{2} p_{i j} 1_{\overline{i j}}(x)
$$

realizes the infimum.

We conclude this section by stating the variational problem of pressure for our setting. We consider the the set of $V_{i}, i=1,2, \ldots, k$ fixed, and we consider a variable set of $W_{i}, i=1,2, \ldots, k$. In the normalized case, the different possible choices of $p_{i}, i=1,2, \ldots, k$, (which means different choices of $\left.W_{i}, i=1,2, \ldots, k\right)$ play here the role of the different Jacobians of possible invariant probabilities (see [15] II.1, and [12]) in Thermodynamic Formalism. In some sense the probabilities $\mu$ can be identified with the Jacobians (this is true at least for Gibbs probabilities of Hölder potentials [17]). The set of Gibbs probabilities for Hölder potentials is dense in the set of invariant probabilities [11].

Let $H: \mathcal{M}_{N} \rightarrow \mathcal{M}_{N}$ be a hermitian operator. We have the following problem. Define $F_{0}: \mathcal{M}_{F} \rightarrow \mathbb{R}$,

$$
F_{0}(\mu):=h_{0}(\mu)-\frac{1}{T} \operatorname{tr}\left(H \rho_{\mu}\right)=\inf _{f \in \mathbb{B}^{+}} \int \log \left(\sum_{i=1}^{k} \frac{f \circ F_{i}}{f}\right) d \mu-\frac{1}{T} \operatorname{tr}\left(H \rho_{\mu}\right),
$$

where $\rho_{\mu}$ is the barycenter of $\mu$, that is, the unique $\rho \in \mathcal{M}_{N}$ such that

$$
l(\rho)=\int_{\mathcal{M}_{N}} l d \mu,
$$

for all $l \in V^{*}$. Then, in order to find the associated Gibbs state we have to find $\hat{\mu} \in \mathcal{M}_{F}$ such that

$$
F_{0}(\hat{\mu})=\sup _{\mu \in \mathcal{M}_{F}} F_{0}(\mu) .
$$

We consider above each $\mu$ which is associated to a possible set of $W_{i}$. 


\section{References}

[1] Baraviera, A., Lardizabal, C. F., A. Lopes, A. O., Terra Cunha, M. A dynamical point of view of Quantum Information: entropy, pressure and Wigner measures. Preprint Arxiv (2009).

[2] Baraviera, A., Lardizabal, C. F., A. Lopes, A. O., Terra Cunha, M. A Thermodynamic Formalism for density matrices in Quantum Information. Preprint Arxiv (2009).

[3] Benatti, F. Dynamics, Information and Complexity in Quantum Systems. Springer Verlag, 2009.

[4] Benenti, G., Casati, G. Strini, G. Principles of Quantum Computation and Information, Vols. I and II, World Scientific, 2007.

[5] Castro, G., Lopes, A. O. KMS States, Entropy and a Variational Principle for Pressure, to appear in Real Analysis Exchange (2009).

[6] Gardiner. C. W., Zoller, P. Quantum Noise, Springer Verlag, 2004.

[7] Gudder, S. Quantum Probability. Academic Press, 1988.

[8] Gudder, S., Schindler, C. Quasi-discrete quantum Markov processes. Annales de l'Institute Henri Poincaré. Vol 56, no. 2, 1992.

[9] Gudder, S. Quantum measure and Integration Theory, preprint (2009).

[10] Lardizabal, C. F. Processos Estocásticos Quânticos, Tese de doutorado Prog. Posgrad. Mat. UFRGS - to appear (2010).

[11] Lopes, A. O. Entropy and Large Deviation. NonLinearity Vol. 3, N. 2, 527-546, 1990.

[12] Lopes, A. An analogy of the charge distribution on Julia sets with the Brownian motion. J. Math. Phys. 30 (9), 1989.

[13] Lopes, A., Oliveira, E. Entropy and variational principles for holonomic probabilities of IFS. Discrete and Continuous Dynamical Systems Vol. 23, N, 3, 937-955 (2009) Series A.

[14] Lozinski, A., Życzkowski, K., Słomczyński, W. Quantum iterated function systems, Physical Review E, Volume 68, 04610, 2003.

[15] Mañé, R. Ergodic Theory, Springer Verlag, 1986.

[16] Nielsen, M., Chuang, I. Quantum computation and quantum information. Cambridge University Press, 2000.

[17] Parry, W., Pollicott, M. Zeta Functions and the Periodic Orbit Structure of Hyperbolic Dynamics. Société Mathématique de France. 187-188, Astérisque, 1990.

[18] Słomczyński, W., Życzkowski, K. Quantum Chaos: an entropy approach. J. Math. Physics, 32 (1), 1994, p. 5674-5700.

[19] Słomczyński, W. Dynamical Entropy, Markov Operators and Iterated Function Systems. Jagiellonian University Press, 2003.

[20] Srinivas, M. D. Foundations of a quantum probability theory. Journal of Math. Phys., Vol. 16, No. 8, 1975. 